\title{
On the molecular theory of aqueous electrolyte solutions. IV. Effects of solvent polarizability
}

\author{
P. G. Kusalika) \\ Research School of Chemistry, The Australian National University, Canberra, Australian Capital Territory, \\ Australia, 2601 \\ G. N. Patey \\ Department of Chemistry, University of British Columbia, Vancouver, British Columbia, \\ Canada, V6T 1 Y6
}

(Received 24 February 1989; accepted 20 September 1989)

This paper describes a new level of theory with which to study model electrolyte solutions with a polarizable solvent. The theory considers the average local electric field experienced by a solvent particle as a function of its separation, $R$, from an ion and is referred to as the $R$ dependent mean-field (RDMF) approximation. Explicit expressions are derived for model solutions consisting of hard-sphere ions immersed in a hard polarizable dipole tetrahedralquadrupole solvent. The lateral solvent fields are shown to cancel a large portion of the field generated by the ionic charge. The RDMF gives rise to an effective spherical ion-solvent potential which will affect the low-concentration limiting behavior of certain thermodynamic properties. The reference hypernetted-chain (RHNC) approximation is solved to obtain RDMF/RHNC results at infinite dilution and low concentration for several model aqueous electrolyte solutions. The ion-ion correlations and thermodynamic quantities such as the mean activity coefficient and partial molar volume of the solute are found to be particularly sensitive to the treatment of the solvent polarizability as this level.

\section{INTRODUCTION}

Recent studies of polar-polarizable fluids using both approximate theories $^{1-6}$ and computer simultions ${ }^{6-10}$ have shown that the many-body interactions due to molecular polarizability are important in determining the equilibrium properties of these systems. The importance of polarization effects in water ${ }^{2,11-14}$ and in electrolyte solutions ${ }^{14,15}$ is now also well established.

In general, the many-body problem of polarizability is difficult to treat in dense liquids and solutions. Fortunately, recent work ${ }^{1-3}$ has demonstrated that in pure fluids it is possible to take into account the influence of many-body interactions due to polarizability through effective pair potentials. The self-consistent mean-field (SCMF) theory ${ }^{2}$ has been shown ${ }^{6,16}$ to be an accurate means of reducing the many-body potential for a fluid of polarizable particles with dipole and tetrahedral-quadrupole moments. For purely dipolar systems the SCMF approximation is equivalent to the $1-R$ theory of Wertheim. ${ }^{3}$ Unlike other methods, however, the SCMF theory uses approximations that are distinctly physical in nature. In paper I of this series ${ }^{17}$ (hereafter referred to as I) we have extended the SCMF expressions to include ion and octupole terms in order to facilitate its application to water-like solvents ${ }^{18}$ (which include multipole moments up to octupole order) and models for aqueous electrolyte solutions. ${ }^{17,19,20}$

In the SCMF theory ${ }^{2,17}$ the pairwise additive potentials which result from the reduction of the many-body interactions of a polarizable system are written in terms of an effective permanent dipole moment, $m_{e}$. This effective dipole moment is an average molecular property of the fluid and

\footnotetext{
") Present address: Department of Chemistry, Dalhousie University, Halifax, Nova Scotia, Canada, B3H $4 \mathrm{~J} 3$.
}

depends upon the polarizability and multipole moments of the model, as well as upon the state parameters of the system. Within the SCMF approximation, systems characterized by $m_{e}$ have the same structural and dielectric properties ${ }^{16}$ as the true polarizable fluid.

The SCMF theory has been used in the study of model aqueous electrolyte solutions at infinite dilution ${ }^{19,21}$ where the effective dipole moment $m_{e}$ must simply be that of the pure solvent. At finite concentration we might expect $m_{e}$ to vary due to the presence of the ions and the resulting changes in the solvent structure. In I, however, it was found that within the framework of the SCMF theory the average local electric field acting on a solvent particle is nearly independent of the salt concentration, at least for the model aqueous electrolyte solutions examined. Hence, to a very good approximation the effective dipole moment can be treated as.a constant equal to the pure solvent value. Nevertheless, we would still expect the average local electric field in the immediate vicinity of an ion to be significantly different from that in the bulk, particularly at low concentration. It would be very interesting to be able to examine the average local electric field experienced by a solvent molecule as a function of its separation, $R$, from an ion. In this paper we will develop a theoretical approach through which the $R$ dependence of the average dipole moment of a solvent can be estimated.

If we consider the case of an ion and a single polarizable solvent particle (i.e., the low-solvent-density limit), then the dipole moment $p$ induced in the molecule will be given by ${ }^{22}$

$$
p=\alpha q / R^{2},
$$

where

$$
\alpha=\frac{1}{3} \operatorname{Tr} \alpha,
$$

$\alpha$ is the molecular polarizability tensor of the solvent parti- 
cle, and $q$ is the charge on the ion. It immediately follows that the interaction between the charge and the induced moment is

$$
u_{q p}(R)=\frac{-\alpha q^{2}}{2 R^{4}} .
$$

However, in solution an ion is surrounded by more than one solvent molecule. These molecules are ordered in some fashion around the ion and this must give rise to changes in the local electric field felt by the solvent particles. At finite concentration the ion is also surrounded by other ions which act to screen its charge and again alter the local field. Thus, we would expect Eqs. (1a) and (1c) to be rather poor approximations in a dense system such as an electrolyte solution. Our aim here is to extend this approach to a dense fluid (i.e., an aqueous electrolyte solution) taking both dielectric and ionic screening into account.

In this article we describe a new level of theory which goes beyond the SCMF approximation. We introduce a more detailed formalism which can be used to estimate the average local field experienced by a solvent particle at a distance $R$ from an ion. As in the previous SCMF theory we take a mean-field approach, that is to say all fluctuations are ignored. The $\boldsymbol{R}$-dependent mean-field (RDMF) theory, as we shall refer to it, yields an effective spherical potential between the ion and the solvent particle at $R$. Moreover, this spherical potential is found to affect the limiting slopes for thermodynamic quantities which depend upon ion-solvent correlations, such as the partial molar volume of the salt.

\section{THE $\boldsymbol{R}$-DEPENDENT MEAN-FIELD THEORY}

The RDMF theory described below is directly applicable only to solutions of spherically symmetric ions, although extensions to more general systems may be possible. In the following explicit derivation we restrict ourselves to a polarizable solvent model with only dipole and quadrupole moments, where the latter is assumed to have tetrahedral symmetry. ${ }^{2}$ We remark that this solvent model has been used extensively in our studies of aqueous electrolyte solutions. ${ }^{17,19,20}$ The present theory is suited to infinite dilution or low concentration and we consider only solutions of a single salt. However, the basic method could be applied to other models or more general solutions. For convenience all particles are treated as hard spheres, where $d_{\alpha}$ is the hardsphere diameter of species $\alpha$ and $d_{\alpha \beta}=\left(d_{\alpha}+d_{\beta}\right) / 2$. The notation employed is consistent with that of the SCMF theory, details of which can be found elsewhere. ${ }^{2,17}$ We also make extensive use of the rotational invariant representation as described in $I$.

In this formulation the problem addressed is that of an ion immersed in a multipolar-polarizable solvent which may or may not contain other ions. The RDMF theory yields expressions for the change in the average local electric field acting on a solvent particle due to the presence of the ion. By considering only the case of a spherically symmetric ion, we are able to take advantage of the fact that from the viewpoint of the ion the surrounding fluid must be isotropic. All additional average fields generated in the surrounding solution due to the presence of the ion must be directed radially at or away from the ion, and hence appear as though they are being produced by additional (screening) charges placed at the center of the ion. Thus, at least at the mean-field level, we need only examine the dependence of the average local field upon $R$, the distance from the ion. We derive expressions for the average local electric field as functions of $R$ by considering the average interaction between the dipole of a solvent particle fixed at a distance $R$ from the ion and all other particles in the system. This average interaction can then be easily related to the average local field.

The electric field experienced by a solvent molecule at a distance $R$ from an ion will, in general, have both ion and solvent components. For the electrolyte solutions considered here, the average local electric field at $R$ is defined by

$$
\left\langle\mathbf{E}_{l}(R)\right\rangle=\left\langle\mathbf{E}_{l D}(R)\right\rangle+\left\langle\mathbf{E}_{l Q}(R)\right\rangle+\left\langle\mathbf{E}_{l I}(R)\right\rangle,
$$

where the first, second, and third terms are the dipolar, quadrupolar, and total ionic field contributions, respectively. For present purposes it is more convenient to express Eq. (2) in the form

$$
\left\langle\mathbf{E}_{l}(R)\right\rangle=\left\langle\mathbf{E}_{l}\right\rangle+\left\langle\Delta \mathbf{E}_{l}(R)\right\rangle,
$$

in which $\left\langle\mathbf{E}_{l}\right\rangle$ is just the average local electric field of the bulk as given by the SCMF theory and $\left\langle\Delta \mathbf{E}_{l}(R)\right\rangle$ is the correction to this term when a solvent particle is at a distance $R$ from an ion. It is clear that $\left\langle\Delta \mathrm{E}_{l}(R)\right\rangle \rightarrow 0$ as $R \rightarrow \infty$. Now at infinite dilution or sufficiently low concentration we can rewrite Eq. (3) as

$$
\left\langle\mathbf{E}_{l}(R)\right\rangle=\left\langle\mathbf{E}_{l}^{0}\right\rangle+\left\langle\Delta \mathbf{E}_{l}(R)\right\rangle,
$$

where $\left\langle\mathbf{E}_{l}^{0}\right\rangle$ is just the average local electric field of the pure solvent. $\left\langle\Delta \mathbf{E}_{l}(R)\right\rangle$ is the change in the bulk field at $R$ due to the presence of the ion and for the present system it is given by

$$
\left\langle\Delta \mathbf{E}_{l}(R)\right\rangle=\left\langle\Delta \mathbf{E}_{l \underline{P}}(R)\right\rangle+\left\langle\Delta \mathbf{E}_{l Q}(R)\right\rangle+\left\langle\Delta \mathbf{E}_{l I}(R)\right\rangle .
$$

We remark that in I it was found that for the model aqueous electrolyte solutions investigated the average local field was essentially independent of salt concentration. Hence, at least for some systems Eq. (4) should be applicable even at higher concentrations.

As in the SCMF theory, we wish to determine the average total (permanent plus induced) dipole moment. In the present context we define

$$
\mathbf{m}^{\prime}(R)=\boldsymbol{\mu}+\boldsymbol{\alpha} \cdot\left\langle\mathbf{E}_{l}(R)\right\rangle,
$$

where $\mathrm{m}^{\prime}(R)$ is the average total dipole moment of a solvent molecule at a distance $R$ from an ion. Using Eq. (3) it clearly follows that

$$
\mathbf{m}^{\prime}(R)=\mathbf{m}^{\prime}+\Delta \mathbf{p}(R),
$$

where

$$
\Delta \mathbf{p}(R)=\alpha \cdot\left\langle\Delta \mathbf{E}_{l}(R)\right\rangle
$$

is the average excess (i.e., due to the presence of the ion) induced dipole moment of a solvent particle at $R$. In our development of explicit expressions for $\left\langle\Delta \mathbf{E}_{l}(R)\right)$ (as described below) we have found it convenient to express $\left\langle\Delta \mathbf{E}_{l}(R)\right\rangle$ in an intermolecular reference frame in which the $z$ axis is along the ion-solvent vector. In the interest of math- 
ematical simplicity it is advantageous to remove the dependence upon the orientation of the solvent molecule and use

$$
\Delta \mathrm{p}(R)=\alpha\left\langle\Delta \mathbf{E}_{l}(R)\right\rangle
$$

instead of Eq. (6c), where $\alpha$ is the average polarizability of the solvent molecule given by Eq. ( $1 \mathrm{~b}$ ). We point out that for the water molecule $\alpha$ is nearly spherically symmetric ${ }^{23}$ and therefore Eq. (7) will be a very good approximation to Eq. (6c). Furthermore, Eq. (7) becomes exact in the limit of large $R$ (i.e., when the ion-solvent correlations become negligible).

In the SCMF theory as applied in Refs. 2 and 17, the effective pairwise additive system is characterized by the effective permanent dipole moment, $m_{e}$. This effective moment is defined as the square root of the mean-square molecular dipole moment and hence is not exactly equal to $m^{\prime}$ in magnitude. Nevertheless, since the present theory is developed within the SCMF context, it is both convenient and consistent to replace $\mathbf{m}^{\prime}$ with $\mathbf{m}_{e}$ in Eq. (6b). We note that for the present water-like solvent the difference between $m^{\prime}$ and $m_{e}$ is in fact very small (i.e., $<2 \%$ ).

The task of the RDMF theory is to take $\left\langle\Delta \mathbf{E}_{l}(R)\right\rangle$ and $\Delta \mathrm{p}(R)$ into account. To this end we now define the additional ion-solvent interaction term due to $\Delta \mathrm{p}(R)$ as

$$
\begin{aligned}
u_{i s}^{\Delta p}(R)= & -\Delta \mathrm{p}(R) \cdot\left\langle\Delta \mathbf{E}_{l I}(R)\right\rangle \\
& -\Delta \mathrm{p}(R) \cdot\left[\left\langle\Delta \mathbf{E}_{l D}(R)\right\rangle+\left\langle\Delta \mathbf{E}_{l Q}(R)\right\rangle\right] \\
& +\frac{1}{2} \Delta \mathbf{p}(R) \cdot\left\langle\Delta \mathbf{E}_{l}(R)\right\rangle .
\end{aligned}
$$

The first term in Eq. ( 8 ) is the interaction between the excess induced moment and the ion field experienced by a solvent molecule at a distance $R$. We remark that at infinite dilution this term is trivially related to Eq. (1c). The second term in Eq. ( 8 ) takes into account the interaction between the excess moment and all the remaining solvent particles surrounding the ion, while the last term is simply the energy of polarization. Since Eq. (7) ensures that $\Delta p(R)$ and $\left\langle\Delta \mathbf{E}_{l}(R)\right\rangle$ are always in the same direction, we immediately have that

$$
u_{i s}^{\Delta p}(R)=-\frac{1}{2} \Delta p(R)\left\langle\Delta E_{l}(R)\right\rangle,
$$

which can also be written in the form

$$
u_{i s}^{\Delta P}(R)=-[\Delta p(R)]^{2} / 2 \alpha .
$$

It is interesting to point out that $u_{i s}^{\Delta p}(R)$ is a spherically symmetric potential which is always attractive relative to infinite separation. Clearly, Eq. (9b) reduces to Eq. (1c) when $\Delta p(R)$ is given by Eq. (1a).

We now have only to determine expressions for each of the terms in Eq. (5) contributing to $\left\langle\Delta \mathbf{E}_{l}(R)\right\rangle$. However, even for the current simplified model this is a non-trivial task. In the present theory the various contributions to $\left\langle\Delta \mathbf{E}_{l}(R)\right\rangle$ are estimated assuming that only pair correlation functions are known. If higher-order correlation functions were available more accurate theories could be derived, but this is clearly not a practical alternative at the present time. We would expect the approximations derived below to be most accurate at long range and at infinite dilution or low salt concentration where many-body correlations will obviously be less important.

First, we examine the simplest case, that of $\left\langle\Delta \mathrm{E}_{l I}(R)\right\rangle$, the average local ion field at a distance $R$ from an ion. We identify three contributions, the first being the direct term, $\left\langle\Delta \mathbf{E}_{l q}(R)\right\rangle$, due to the charge on the ion. The other two terms, $\left\langle\Delta \mathbf{E}_{l_{+}}(R)\right\rangle$ and $\left\langle\Delta \mathbf{E}_{l-}(R)\right\rangle$, are essentially screening terms and are due to all other positive and negative ions in the system, respectively. Therefore, we write

$$
\left\langle\Delta \mathbf{E}_{l l}(R)\right\rangle=\left\langle\Delta \mathbf{E}_{l q}(R)\right\rangle+\left\langle\Delta \mathbf{E}_{l+}(R)\right\rangle+\left\langle\Delta \mathbf{E}_{l_{-}}(R)\right\rangle .
$$

It is obvious [cf. Eq. (1a)] that

$$
\left\langle\Delta E_{l q}(R)\right\rangle=q_{i} / R^{2},
$$

where $q_{i}$ is the charge on the ion (which we have labeled $i$ ) and $\left\langle\Delta \mathbf{E}_{l q}(R)\right\rangle$ will always be directed along the vector joining the ion $i$ and the solvent particle at $R$.

In order to determine the two remaining terms in Eq. (10) we first examine the average interaction between the dipole moment of a solvent particle at $R$, which we refer to as the reference particle, and all other ions $j$ (see Fig. 1). As a consequence of the $C_{2 V}$ symmetry of the solvent molecule we know that the average orientation of the effective dipole moment of the reference solvent particle will be in the direction of $\left\langle\Delta \mathbf{E}_{l q}(R)\right\rangle$ and that all other orientations must average to zero. Moreover, for the present system $\left\langle\Delta \mathbf{E}_{l}(R)\right\rangle$, and hence $\Delta \mathrm{p}(R)$, will be nonzero only along the ion-solvent vector. Thus, using Eq. (6b) and ignoring fluctuations, we can define

$$
m^{\dagger}(R)=m_{e}\left\langle\cos \theta_{i s}(R)\right\rangle+\Delta p(R)
$$

as the average projection of $\mathrm{m}^{\prime}(R)$ onto the ion-solvent vector, where

$$
\left\langle\cos \theta_{+s}(R)\right\rangle=h_{00 ;+s}^{011}(R) / 3 g_{+s}(R)
$$

and

$$
\left\langle\cos \theta_{-s}(R)\right\rangle=-h_{00 ;-s}^{011}(R) / 3 g_{-s}(R)
$$

are defined in I. It is sufficient here to state simply that $\theta_{i s}$ gives the angle between $\mathbf{m}_{e}$ and $\mathbf{r}_{i s}=\mathbf{r}_{s^{*}}-\mathbf{r}_{i}$ such that $\cos \theta_{i s}$ is positive when $\mathbf{m}_{e}$ is favorably aligned with $\left\langle\Delta \mathbf{E}_{l q}(R)\right\rangle$. Thus $\mathrm{m}^{\dagger}(R)$ is the projection of $\mathrm{m}^{\prime}(R)$ lying in the direction of $\left\langle\Delta \mathbf{E}_{l q}(R)\right\rangle$. Clearly, $\mathrm{m}^{\dagger}(R)$ contains contributions due to both the effective permanent dipole moment, $\mathrm{m}_{e}$, and the average excess induced moment, $\Delta p(R)$. We can now obtain

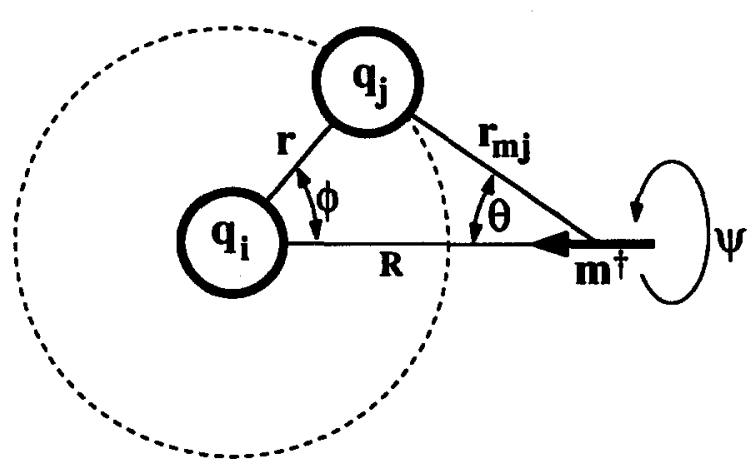

FIG. 1. An illustration of the method used in determining $\left\langle\Delta \mathbf{E}_{I I}(R)\right\rangle$. The case where $q_{i}$ is negative is shown. The dipole moment $\mathrm{m}^{+}$, located at a distance $\boldsymbol{R}$ from the ion $i$, is that of the reference solvent particle. 
an expression for the interaction between $\mathrm{m}^{\dagger}(R)$ and a spherical shell of ions at a distance $r$ from $q_{i}$, which we have only to integrate (i.e., sum over shells) in order to determine a relationship for the total interaction. In Fig. 1 we have illustrated the problem as we have framed it, as well as indicating all the variables used in the following derivation.

From Eqs. (29) and (30) of I it is easy to show that the interaction between $\mathrm{m}^{\dagger}(R)$ and $q_{j}$ is given by

$$
u_{m j}(12)=\left[m^{\dagger}(R) q_{j} / r_{m j}^{2}\right] \Phi_{00}^{101}\left(\mathbf{\Omega}_{1}, \mathbf{\Omega}_{2}, \hat{\mathbf{r}}_{m j}\right),
$$

when we take $f^{m n l}=l ! /\left(\begin{array}{ccc}m & n \\ 0 & l\end{array}\right)$ (which we employ exclusively in the present article). Following the conventions given in Fig. 1 and those for $\mathrm{m}^{\dagger}(R)$, and using an explicit form for the rotational invariant (see Appendix B of Ref. 1), we rewrite Eq. (14) in the form

$$
u_{m j}(12)=-\operatorname{sgn}\left(q_{i}\right)\left[m^{\dagger}(R) q_{j} / r_{m j}^{2}\right] \cos \theta,
$$

where the sgn function equals 1 if $q_{i}$ is positive and -1 if $q_{i}$ is negative. From the law of $\operatorname{cosines}^{24}$ we have relationships for $r_{m j}^{2}$ and $\cos \theta$ in terms of $R, r$, and $\phi$ which, when substituted into Eq. (15), yield

$$
\begin{aligned}
u_{m j}(R, r, \phi)= & -\operatorname{sgn}\left(q_{i}\right) m^{\dagger}(R) q_{j} \\
& \times \frac{R-r \cos \phi}{\left(r^{2}+R^{2}-2 r R \cos \phi\right)^{3 / 2}} .
\end{aligned}
$$

Then the average interaction potential between $\mathrm{m}^{\dagger}(R)$ and the spherical shell of ions (positive or negative) of thickness $d r$ at a distance $r$ from $q_{i}$ is

$$
U_{m j}^{0}(R, r)=\rho_{j}\left[\int g_{m j}(R, r, \phi, \psi) u_{m j}(R, r, \phi) d A\right] d r,
$$

in which the element of area

$$
d A=r^{2} \sin \phi d \phi d \psi \text {. }
$$

The limits of integration for $d \psi$ are 0 to $2 \pi$, while for $d \phi$ they are $\phi_{m}$ to $\pi$, where

$$
\phi_{m}=\left\{\begin{array}{cl}
0 & \text { if }|r-R|>d_{j s} \\
\cos ^{-1}\left[\left(r^{2}+R^{2}-d_{j s}^{2}\right) / 2 r R\right] & \text { if }|r-R|<d_{j s}
\end{array}\right.
$$

guarantees that the ion $j$ and the reference solvent do not interpenetrate.

Now, in principle, we do not know $g_{m j}(R, r, \phi, \psi)$. However, if we take the distribution of ions to be uniform in the spherical shell at $r$, and if we also assume it to be independent of $R$, the position of the reference solvent, then we can write

$$
g_{m j}(R, r, \phi, \psi)=g_{i j}(r),
$$

where $g_{i j}(r)$ is just the ion-ion radial distribution function. We would expect this to become a reasonable approximation at large $r$. Inserting Eqs. (16) and (19) into Eq. (17a) and integrating over $d \psi$, we obtain

$$
\begin{aligned}
U_{m j}^{0}(R, r)= & -2 \pi r^{2} \rho_{j} g_{i j}(r) d r \operatorname{sgn}\left(q_{i}\right) q_{j} m^{\dagger}(R) \\
& \times \int_{\phi_{m}}^{\pi} \frac{(R-r \cos \phi) \sin \phi}{\left(r^{2}+R^{2}-2 r R \cos \phi\right)^{3 / 2}} d \phi .
\end{aligned}
$$

Then carefully integrating Eq. (20) (using standard forms for the trigonometric integral which may be found in tables $^{24}$ ) and simplifying yields the expressions

$$
\begin{aligned}
& U_{m j}^{0}(R, r)=0 \text { for } r \geqslant R+d_{j s}, \\
& U_{m j}^{0}(R, r)=-.2 \pi r^{2} \rho_{j} g_{i j}(r) \operatorname{sgn}\left(q_{i}\right) \frac{q_{j} m^{\dagger}(R)}{R^{2}}\left(1+\frac{R^{2}-r^{2}-d_{j s}^{2}}{2 r d_{j s}}\right) d r \quad \text { for } R-d_{j s} \leqslant r \leqslant R+d_{j s},
\end{aligned}
$$

and

$$
U_{m j}^{0}(R, r)=-4 \pi r^{2} \rho_{j} g_{i j}(r) \operatorname{sgn}\left(q_{i}\right) \frac{q_{j} m^{\dagger}(R)}{R^{2}} d r \text {, for } r \leqslant R-d_{j s} .
$$

We remark that Eqs. (21) are consistent with basic electrostatic theory ${ }^{25}$ which states that from an internal point of view a spherical shell of charge is electrically nonexistent [cf. Eq. (21a)], and from an external point of view it is equivalent to a point charge whose charge is equal to that contained in the shell [cf. Eq. (21c)].

The total average interaction potential, $U_{m j}(R)$, between $\mathrm{m}^{\dagger}(R)$ and all other ions $j$ is found by simply integrating Eqs. (21) over all values of $r$. The ions $i$ and $j$ are not allowed to interpenetrate so we write

$$
U_{m j}(R)=\int_{d_{i j}}^{\infty} U_{m j}^{0}(R, r),
$$

and inserting Eqs. (21) we obtain the necessary result

$$
U_{m j}(R, r)=-4 \pi \rho_{j} \operatorname{sgn}\left(q_{i}\right) \frac{q_{j} m^{\dagger}(R)}{R^{2}}\left[\int_{d_{i j}-d_{j s}} r^{2} g_{j j}(r) d r+\frac{1}{2} \int_{R-d_{j s}}^{R+d_{j s}} r^{2} g_{i j}(r)\left(1+\frac{R^{2}-r^{2}-d_{j s}^{2}}{2 r d_{j s}}\right) d r\right],
$$

where we recall that $j=+$ or - . Now for the present system it is clear that $\left\langle\Delta \mathbf{E}_{l j}(R)\right\rangle$ and $\mathrm{m}^{\dagger}(R)$ are parallel, and consequently we also have that

$$
U_{m j}(R)=-m^{\dagger}(R)\left\langle\Delta E_{l j}(R)\right\rangle .
$$

Together Eqs. (23) and (24) provide an expression for $\left\langle\Delta \mathbf{E}_{l}(R)\right\rangle$ for $j=+,-$ which, when combined with Eqs. (10) and (11), gives the desired relationship 


$$
\left\langle\Delta E_{l I}(R)\right\rangle=\frac{\operatorname{sgn}\left(q_{i}\right)}{R^{2}}\left(q_{i}+\sum_{j=+,-} 4 \pi \rho_{j} q_{j}\left\{\int_{d_{i j}}^{R-d_{j s}} r^{2} g_{i j}(r) d r+\frac{1}{4 d_{j s}} \int_{R-d_{j s}}^{R+d_{j s}} r g_{i j}(r)\left[R^{2}-\left(r-d_{j s}\right)^{2}\right] d r\right\}\right),
$$

where $\left\langle\Delta \mathbf{E}_{I I}(R)\right\rangle$ is always directed along the ion-solvent vector. We note that in obtaining Eq. (25) we have multiplied Eq. (11) by $\operatorname{sgn}\left(q_{i}\right)$ to ensure that $\left\langle\Delta \mathbf{E}_{l q}(R)\right\rangle$ has the same directional sense as $\left\langle\Delta \mathbf{E}_{l j}(R)\right\rangle$.

We now turn our attention to $\left\langle\Delta \mathbf{E}_{l D}(R)\right\rangle$. If we consider an ion in a polar solvent of $C_{2 V}$ symmetry, as is the case here, we find that on average all the dipole moments point either directly at or directly away from the ion [cf. Eq. (12)]. We shall refer to these average projections as being lateral. Clearly, all lateral dipoles in the same spherical shell will mutually repel one another. It is this contribution to the local dipole field which we examine.

The lateral dipole field, and hence $\left\langle\Delta \mathbf{E}_{l D}(R)\right\rangle$ in the present theory, is determined in a manner quite similar to that employed for $\left\langle\Delta \mathrm{E}_{I I}(R)\right\rangle$. Again, it is only the average projection, $\mathrm{m}^{\dagger}(R)$, of the total dipole moments that needs to be considered. In order to derive an expression for $\left\langle\Delta E_{l D}(R)\right\rangle$ we first examine the interaction between the reference dipole, $\mathbf{m}^{\dagger}(R)$, and all spherical shells of dipole moments, $\mathrm{m}^{\dagger}(r)$, at a distance $r$ from the ion $i$. In Fig. 2 we have illustrated the situation being considered along with all the necessary variables.

From Eqs. (29) and (30) of I it can be shown that the interaction between $\mathrm{m}^{\dagger}(r)$ and $\mathrm{m}^{\dagger}(R)$ is given by ${ }^{2}$

$$
u_{m m}(12)=-\left[m^{\dagger}(r) m^{\dagger}(R) / r_{m m}^{3}\right] \Phi_{\mathrm{oO}}^{112}\left(\mathbf{\Omega}_{1}, \mathbf{\Omega}_{2}, \hat{\mathbf{r}}_{m m}\right) .
$$

The rotational invariant can be written in the form ${ }^{1}$

$$
\begin{aligned}
\Phi_{00}^{112}\left(\mathbf{\Omega}_{1}, \mathbf{\Omega}_{2}, \hat{\mathbf{r}}\right)= & 2 \cos \theta_{1} \cos \theta_{2} \\
& -\sin \theta_{1} \sin \theta_{2} \cos \left(\phi_{1}-\phi_{2}\right),
\end{aligned}
$$

where the angles $\theta_{1}$ and $\theta_{2}$ are indicated in Fig. 2, and $\phi_{1}$ and $\phi_{2}$ are the azimuthal angles. It is obvious from Fig. 2 that $\phi_{1}$ and $\phi_{2}$ must always be equal. The law of sines and the law of $\operatorname{cosines}^{24}$ provide relationships for $r_{m m}^{2}, \cos \theta_{1}, \cos \theta_{2}, \sin \theta_{1}$, and $\sin \theta_{2}$ in terms of $R, r$, and $\phi$ which can be substituted

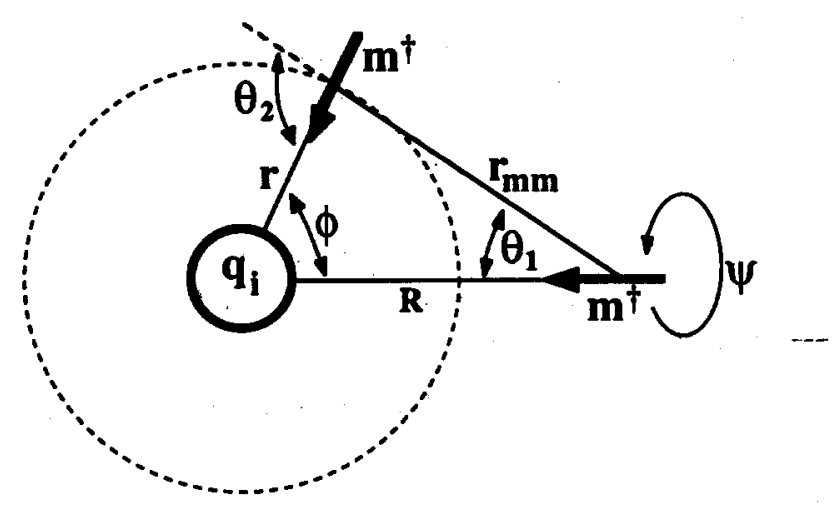

FIG. 2. An illustration of the method used in determining $\left\langle\Delta \mathbf{E}_{l D}(R)\right\rangle$. The case where $q_{i}$ is negative is shown. into Eq. (27). Then combining the result obtained with Eq. (26) yields

$$
\begin{aligned}
u_{m m}(R, r, \phi)= & \frac{m^{\dagger}(r) m^{\dagger}(R)}{\left(r^{2}+R^{2}-2 r R \cos \phi\right)^{5 / 2}} \\
& \times\left[3 r R-2\left(r^{2}+R^{2}\right) \cos \phi+r R \cos ^{2} \phi\right] .
\end{aligned}
$$

We remark that this expression is invariant to $\operatorname{sgn}\left(q_{i}\right)$ since both $\mathrm{m}^{\dagger}(r)$ and $\mathrm{m}^{\dagger}(R)$ reverse direction if the charge on the ion is reversed [i.e., both contribute a factor of $\operatorname{sgn}\left(q_{i}\right)$ ].

We express the average lateral dipole-dipole interaction, $U_{m m}^{\text {lat }}(R, r)$, between $\mathrm{m}^{\dagger}(R)$ and the spherical shell of dipoles at a distance $r$ from the same ion as

$U_{m m}^{\text {lat }}(R, r)=\rho_{s}\left[\int g_{s s}(R, r, \phi, \psi) u_{m m}(R, r, \phi) d A\right] d r$,

where $d A$ is given by Eq. (17b). The limits of integration are still 0 to $2 \pi$ for $d \psi$ and $\phi_{m}$ to $\pi$ for $d \phi$, as was the case for $\left\langle\Delta \mathrm{E}_{I I}(R)\right\rangle$, but now

$\phi_{m}=\left\{\begin{array}{cl}0 & \text { if }|r-R| \geqslant d_{s} \\ \cos ^{-1}\left[\left(r^{2}+R^{2}-d_{s}^{2}\right) / 2 r R\right] & \text { if }|r-R| \leqslant d_{s}\end{array}\right.$.

Again, we assume that $g_{s s}(R, r, \phi, \psi)$ is spherically symmetric (i.e., the solvents are uniformly distributed in the shell) and independent of $R$ so we take

$$
g_{s s}(R, r, \phi, \psi)=g_{i s}(r) .
$$

We now substitute Eqs. (28) and (31) into (29) and integrate, making use of standard tables of trigonometric integrals. ${ }^{24}$ After considerable manipulation it can be shown that

$$
U_{m m}^{\text {lat }}(R, r)=0 \text { for }|r-R| \geqslant d_{s}
$$

and

$$
\begin{aligned}
U_{m m}^{\text {lat }}(R, r) & =2 \pi r^{2} \rho_{s} g_{i s}(r) \frac{m^{\dagger}(r) m^{\dagger}(R)}{d_{s}^{3}} \\
& \times\left[1-\left(\frac{r^{2}+R^{2}-d_{s}^{2}}{2 r R}\right)^{2}\right] d r \text { for }|r-R| \leqslant d_{s} .
\end{aligned}
$$

The total average dipole interaction $U_{m m}(R)$ between $\mathrm{m}^{\dagger}(R)$ and all other dipole moments is found by integrating Eqs. (32) over all values of $r$, although only those shells for which $|r-R|<d_{s}$ contribute. We obtain the expression

$$
\begin{aligned}
U_{m m}(R)= & \frac{\pi \rho_{s} m^{\dagger}(R)}{2 d_{s}^{3} R^{2}} \int_{R-d_{s}}^{R+d_{s}} m^{\dagger}(r) g_{i s}(r) \\
& \times\left[(2 r R)^{2}-\left(r^{2}+R^{2}-d_{s}^{2}\right)^{2}\right] d r .
\end{aligned}
$$

Finally, taking advantage of the fact that all the average projected moments $\mathrm{m}^{\dagger}(r)$ are directed along the ion-solvent 
vector and distributed uniformly about the ion, it must follow that the only nonzero component of the total average lateral dipole field will be in the direction of $\mathrm{m}^{\dagger}(R)$. Thus analogous to Eq. (24) we write

$$
U_{m m}(R)=-m^{\dagger}(R)\left\langle\Delta E_{l D}(R)\right\rangle .
$$

Combining Eqs. (33) and (34) and inserting the results from Eqs. (12) and (13) yields the relationship

$$
\begin{aligned}
& \left\langle\Delta E_{l D}(R)\right\rangle \\
& =\frac{\pi \rho_{s}}{2 d_{s}^{3} R^{2}} \int_{R-d_{s}}^{R+d_{s}}\left[\frac{m_{e} h_{00 ; i s}^{011}(r)}{3 \operatorname{sgn}\left(q_{i}\right)}+\Delta p(r) g_{i s}(r)\right] \\
& \quad \times\left[\left(r^{2}+R^{2}-d_{s}^{2}\right)^{2}-(2 r R)^{2}\right] d r .
\end{aligned}
$$

Inspection of Eq. (35) reveals two distinct contributions to $\left\langle\Delta \mathbf{E}_{l D}(R)\right\rangle$, one due only to $\mathrm{m}_{e}$ and the other due to $\Delta \mathrm{p}(R)$. This separation will prove useful in discussions below.

$\left\langle\Delta \mathbf{E}_{l Q}(R)\right\rangle$ is determined in a fashion very similar to that used for $\left\langle\Delta \mathbf{E}_{l D}(R)\right\rangle$. We again consider only the contribution to $\left\langle\Delta \mathbf{E}_{Q}(R)\right\rangle$ due to lateral fields, where these lateral fields are a consequence of the average projections of the quadrupole moments of the solvent particles around the ion $i$. These projections are analogous to the projections $\mathbf{m}^{\dagger}(r)$ and are defined below. We emphasize here that this derivation applies strictly to quadrupole moments of tetrahedral symmetry, as defined in Refs. 2 and 17. Geometrically, such a quadrupole moment can be represented by a charge distribution in which two positive and two negative charges of

$\boldsymbol{\Phi}^{123}\left(\mathbf{\Omega}_{1}, \mathbf{\Omega}_{2}, \hat{\mathbf{r}}\right)=\sqrt{6}\left\{3 \cos \beta_{1} \sin ^{2} \beta_{2} \cos 2 \gamma_{2}+2 \sin \beta_{1} \sin \beta_{2}\right.$ tion that has a quadrupole of tetrahedral symmetry as its
As in the case of $\left\langle\Delta \mathbf{E}_{l D}(R)\right\rangle$, we need to determine the average interaction between all average projections, $\mathbf{Q}^{\dagger}(r)$, of the quadrupole moments in a spherical shell at a distance $r$ from the ion $i$ and the dipole moment $\mathrm{m}^{\dagger}(R)$. In Fig. 3 we have represented this geometrical problem, indicating the variables pertinent to the following derivation. However, in order to proceed we must first define $\mathbf{Q}^{\dagger}(r)$. It follows from Eqs. (29) - (31) of I and from Eq. (38a) that the interaction between an ion and a quadrupole of tetrahedral symmetry is given by ${ }^{21}$

$$
u_{i Q}(12)=\frac{q_{i} Q_{T}}{r^{3}} \sin ^{2} \beta_{2} \cos 2 \gamma_{2} .
$$

It is the most energetically favorable orientation with respect to the ion $i$ which is pictured in Fig. 3(a). This orientation of the quadrupole moment is the only one that does not average to zero for molecules of $C_{2 V}$ symmetry, so it is onto this orientation that we determine the average projection of the quadrupole moment. It is clear from Eq. (39) that for $Q_{T}$ positive, which is the case for the present water-like solvent, ${ }^{17}$ this projection corresponds to

$$
\beta \frac{I}{2}=\pi / 2
$$

and $\left.\left[\cos \beta_{2} \cos 2 \gamma_{2} \cos \left(\alpha_{1}-\alpha_{2}\right)+\sin 2 \gamma_{2} \sin \left(\alpha_{1}-\alpha_{2}\right)\right]\right\}$.

and

$$
\begin{aligned}
\Phi^{123} & \left(\boldsymbol{\Omega}_{1}, \mathbf{\Omega}_{2}, \hat{\mathbf{r}}\right) \\
= & \sqrt{6}\left\{5\left[\left(\hat{\mathbf{x}}_{2} \cdot \hat{\mathbf{r}}_{12}\right)^{2}-\left(\hat{\mathbf{y}}_{2} \cdot \hat{\mathbf{r}}_{12}\right)^{2}\right]\left(\hat{\mathbf{z}}_{1} \cdot \hat{\mathbf{r}}_{12}\right)\right. \\
& \left.-2\left[\left(\hat{\mathbf{x}}_{2} \cdot \hat{\mathbf{z}}_{1}\right)\left(\hat{\mathbf{x}}_{2} \cdot \hat{\mathbf{r}}_{12}\right)-\left(\hat{\mathbf{y}}_{2} \cdot \hat{\mathbf{z}}_{1}\right)\left(\hat{\mathbf{y}}_{2} \cdot \hat{\mathbf{r}}_{12}\right)\right]\right\},
\end{aligned}
$$

where $\hat{\mathbf{x}}, \hat{\mathbf{y}}$, and $\hat{\mathbf{z}}$ are the appropriate molecular fixed unit vectors and $\hat{\mathbf{r}}_{12}=\mathbf{r}_{12} /\left|\mathbf{r}_{12}\right|$. Then using the definitions of the Euler angles $\alpha, \beta, \gamma$ and the rotation matrix [see Eq. (39)] of Ref. 27, it can be shown that

$$
\boldsymbol{\Phi}^{022}\left(\boldsymbol{\Omega}_{1}, \boldsymbol{\Omega}_{2}, \hat{\mathbf{r}}\right)=\sqrt{6} \sin ^{2} \beta_{2} \cos 2 \gamma_{2}
$$

and

equal magnitude have been placed at the corners of a square lying perpendicular to the $z$ axis such that like charges are lowest-order moment.

We start by defining the functions

$\Phi^{022}\left(\boldsymbol{\Omega}_{1}, \mathbf{\Omega}_{2}, \hat{\mathbf{r}}\right)=\Phi_{02}^{022}\left(\mathbf{\Omega}_{1}, \mathbf{\Omega}_{2}, \hat{\mathbf{r}}\right)+\Phi_{0-2}^{022}\left(\mathbf{\Omega}_{1}, \mathbf{\Omega}_{2}, \hat{\mathbf{r}}\right)$

and

analogous to those used in Refs. 2 and 21, which can be written in the explicit forms ${ }^{26,27}$

$$
\Phi^{022}\left(\boldsymbol{\Omega}_{1}, \mathbf{\Omega}_{2}, \hat{\mathbf{r}}\right)=\sqrt{6}\left[\left(\hat{\mathbf{x}}_{2} \cdot \hat{\mathbf{r}}_{12}\right)^{2}-\left(\hat{\mathbf{y}}_{2} \cdot \hat{\mathbf{r}}_{12}\right)^{2}\right]
$$

\section{$\Gamma$}

$$
\cos 2 \gamma_{2}^{I}=-\operatorname{sgn}\left(q_{i}\right),
$$

where the superscript $I$ indicates the angles as defined for the ion reference frame [see Fig. 3(b)]. We remark that $u_{i Q}(12)$ is independent of $\alpha_{2}^{I}$ and therefore $Q^{\dagger}(r)$ is allowed to spin freely about the $z_{I}$ axis. We then write that

$$
Q^{\dagger}(r)=\frac{-Q_{T}}{\operatorname{sgn}\left(q_{i}\right)}\left\langle\Phi^{022}(r)\right\rangle,
$$

where $\left\langle\Phi^{022}(r)\right\rangle$ is just the average value of $\Phi^{022}\left(\Omega_{1}, \Omega_{2}, \hat{\mathbf{r}}\right)$ for a solvent particle at a distance $r$ from an ion. Using an explicit expression for $\left\langle\Phi^{022}(r)\right\rangle$ [obtained by combining Eq. (36a) and Eq. (2.87) of Ref. 28], Eq. (41) becomes

$$
Q^{\dagger}(r)=\frac{-Q_{T}}{\operatorname{sgn}\left(q_{i}\right)} \frac{8 h_{02 ; i s}^{022}(r)}{5 g_{i s}(r)} .
$$

Now the interaction $u_{m Q}(12)$ between the dipole moment $\mathrm{m}^{\dagger}(R)$ and the quadrupole moment $\mathbf{Q}^{\dagger}(r)$ can be expressed in the form ${ }^{2}$

$$
u_{m Q}(12)=\frac{m^{\dagger}(R) Q^{\dagger}(r)}{\sqrt{6} r_{m Q}^{4}} \Phi^{123}\left(\mathbf{\Omega}_{1}, \mathbf{\Omega}_{2}, \hat{\mathbf{r}}_{m Q}\right) .
$$

Unfortunately, the function $\Phi^{123}\left(\Omega_{1}, \Omega_{2}, \hat{r}\right)$, as given by Eq. (38b), is not expressed in terms of the angles shown in Fig. 3 . Thus, we must first rewrite Eq. (38b) as a function of these 
angles (i.e., $\alpha_{2}^{I}, \beta_{2}^{I}, \gamma_{2}^{I}, \beta_{1}, \omega$ ). In Appendix A we briefly outline how this may be done (a more complete description can be found in Appendix $C$ of Ref. 28) to give

$\Phi^{123}\left(\Omega_{1}, \Omega_{2}, \hat{\mathbf{r}}\right)=\sqrt{6}\left[3 \cos \beta_{1}\left(\cos ^{2} \omega-\sin ^{2} \alpha_{2}^{I} \sin ^{2} \omega\right)+2 \sin \beta_{1} \sin \omega \cos \omega\left(1+\sin ^{2} \alpha_{2}^{I}\right)\right] \cos 2 \gamma_{2}^{I}$.

Finally, we replace $\beta_{1}$ by $\theta_{1}\left(\theta_{1}+\pi\right.$ if $q_{i}$ is positive) and eliminate the $\cos 2 \gamma_{2}^{I}$ dependence in Eq. (44) by noting that the products

$$
\cos \beta_{1} \cos 2 \gamma_{2}^{I}=\cos \theta_{1}
$$

and

$$
\sin \beta_{1} \cos 2 \gamma_{2}^{I}=\sin \theta_{1}
$$

are independent of the sign of $q_{i}$. Combining Eqs. (43), (44), and (45) yields

$u_{m Q}(12)$

$$
\begin{aligned}
= & \frac{m^{\dagger}(R) Q^{\dagger}(r)}{r_{m Q}^{4}}\left[3 \cos \theta_{1}\left(\cos ^{2} \omega-\sin ^{2} \alpha_{2}^{I} \sin ^{2} \omega\right)\right. \\
& \left.+2 \sin \theta_{1} \sin \omega \cos \omega\left(1+\sin ^{2} \alpha_{2}^{I}\right)\right] .
\end{aligned}
$$

We now recall that $\mathbf{Q}^{\dagger}(r)$ is allowed to spin freely about the $z_{1}$ axis, and therefore we cannot specify the angle $\alpha_{2}^{I}$. How- ever, the $\alpha_{2}^{I}$ dependence can be removed from Eq. (46) by taking advantage of the fact that all angles $\alpha_{2}^{I}$ are equally probable (at the present level of theory) and simply angle averaging over them. After performing the required integration we have the result

$$
\begin{aligned}
u_{m Q}(12)= & \frac{3 m^{\dagger}(R) Q^{\dagger}(r)}{r_{m Q}^{4}}\left[\cos \theta_{1}\left(\cos ^{2} \omega-\frac{1}{2} \sin ^{2} \omega\right)\right. \\
& \left.+\sin \theta_{1} \sin \omega \cos \omega\right] .
\end{aligned}
$$

As above we employ the law of sines and the law of cosines $^{24}$ in order to express the functions of $\theta_{1}, \omega$, and $r_{m e}$ appearing in Eq. (47) as functions of $R, r$, and $\phi$. The average lateral quadrupole-dipole interaction, $U_{m Q}^{\text {lat }}(R, r)$, is defined by an expression analogous to Eq. (29). Then inserting Eq. (47) together with the approximation (31) and integrating over $d \psi$, we obtain

$$
\begin{aligned}
U_{m Q}^{\text {lat }}(R, r)= & 6 \pi r^{2} \rho_{s} g_{\text {is }}(r) m^{\dagger}(R) Q^{\dagger}(r) d r \\
& \times \int_{\phi_{m}}^{\pi} \frac{2 r^{2} R-R^{3} / 3-\left(\frac{5}{2} r R^{2}+r^{3}\right) \cos \phi+\left(r^{2} R+\frac{3}{2} R^{3}\right) \cos ^{2} \phi-\left(r R^{2} / 2\right) \cos ^{3} \phi}{\left(r^{2}+R^{2}-2 r R \cos \phi\right)^{7 / 2}} \sin \phi d \phi,
\end{aligned}
$$

where $\phi_{m}$ is given by Eq. (30). The integration of Eq. (48) requires considerable effort, but with the aid of tables of standard trigonometric integrals ${ }^{24}$ and after much simplification the result can be written as

$$
U_{m Q}^{\text {lat }}(R, r)=0 \text { for }|r-R| \geqslant d_{s}
$$

and

$U_{m Q}^{\text {lat }}(R, r)=3 \pi r^{2} \rho_{s} g_{i s}(r) \frac{m^{\dagger}(R) Q^{\dagger}(r)}{d_{s}^{5}}\left[1-\left(\frac{r^{2}+R^{2}-d_{s}^{2}}{2 r R}\right)^{2}\right]\left(\frac{r^{2}-R^{2}+d_{s}^{2}}{2 r}\right) d r$ for $|r-R| \leqslant d_{s}$.

We remark that these expressions bear a striking similarity to Eqs. (32), the lateral dipole result.

The total average lateral quadrupole-dipole interaction, $U_{m Q}(R)$, is found by integrating Eqs. (49) over all values of $r$. Arguments very similar to those used for $\left\langle\Delta \mathbf{E}_{l D}(R)\right\rangle$ can be used to show that $\left\langle\Delta \mathbf{E}_{l Q}(R)\right\rangle$ will also only be nonzero in the direction of $\mathbf{m}^{\dagger}(R)$, so we can write
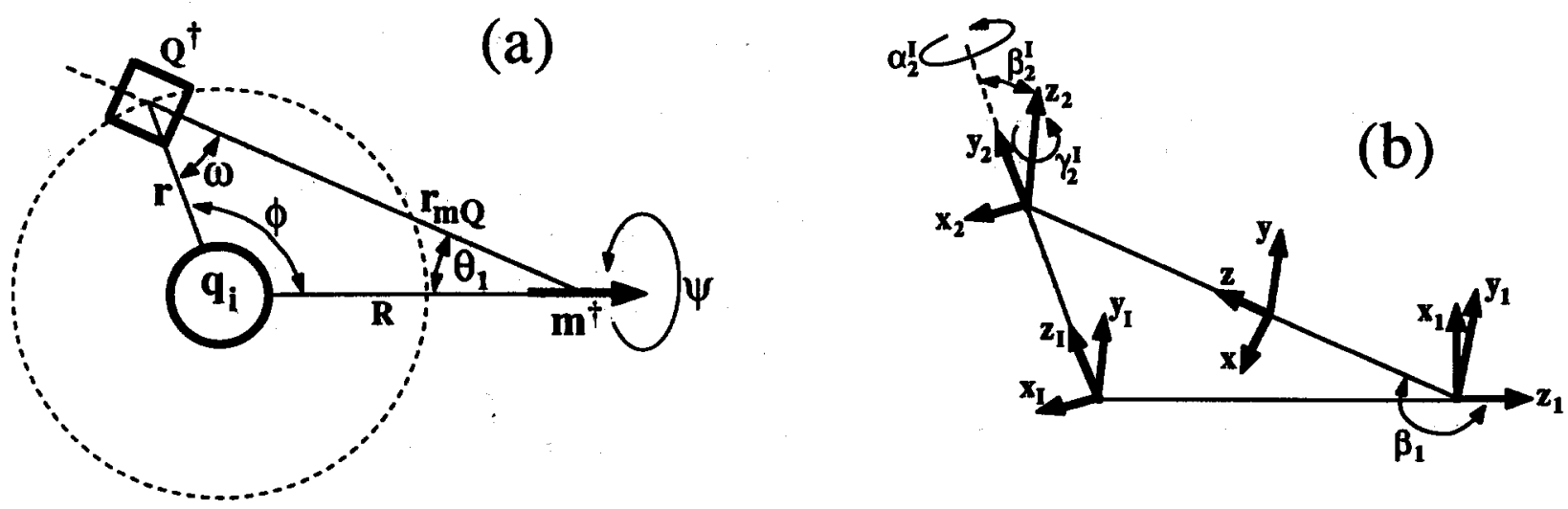

FIG. 3. An illustration of the method used in determining $\left\langle\Delta \mathbf{E}_{l q}(R)\right)$. The case where $q_{\text {, }}$ is positive is shown. For clarity the various reference frames and their rotations are given separately in (b). 


$$
U_{m Q}(R)=-m^{\dagger}(R)\left\langle\Delta E_{l Q}(R)\right\rangle .
$$

Finally, combining these two expressions for $U_{m Q}(R)$ and inserting Eq. (42) gives the desired result

$$
\left\langle\Delta E_{l Q}(R)\right\rangle=\frac{3 \pi \rho_{s} Q_{T}}{5 \operatorname{sgn}\left(q_{i}\right) d_{s}^{5} R^{2}} \int_{R-d_{s}}^{R+d_{s}} h_{02 ; i s}^{022}(r) / r\left[(2 r R)^{2}-\left(r^{2}+R^{2}-d_{s}^{2}\right)^{2}\right]\left(r^{2}-R^{2}+d_{s}^{2}\right) d r .
$$

It is interesting to point out that both $\left\langle\Delta E_{l D}(R)\right\rangle$ and $\left\langle\Delta E_{l Q}(R)\right\rangle$, as given by Eqs. (35) and (51), respectively, have an apparent $1 / R^{2}$ dependence.

We now have expressions for all three terms contributing to $\left\langle\Delta \mathbf{E}_{l}(R)\right\rangle$. Given the necessary projections of the pair correlation functions, $\left\langle\Delta \mathbf{E}_{l I}(R)\right\rangle$ and $\left\langle\Delta \mathbf{E}_{l Q}(R)\right\rangle$ can be evaluated directly. However, $\left\langle\Delta \mathbf{E}_{l D}(R)\right\rangle$ must be solved iteratively since it depends upon $\Delta p(R)$ [cf. Eq. (35)], which in turn depends upon $\left\langle\Delta E_{l D}(R)\right\rangle$. Once $\left\langle\Delta \mathbf{E}_{l}(R)\right\rangle$ has been determined, Eqs. (7) and (9) immediately give the effective ion-solvent potential, $u_{i s}^{\Delta p}(R)$, for the RDMF theory.

We recall that $u_{i s}^{\Delta p}(R)$ is a spherically symmetric term in the ion-solvent interaction potential. Furthermore, within the hypernetted-chain (HNC) approximation it is possible to show [cf. Eq. (B3) of Ref. 29] that as $\rho_{2} \rightarrow 0$ and $r \rightarrow \infty$,

$$
c_{00 ; i s}^{000}(r) \rightarrow \frac{1}{6}\left[h_{00 ; i s}^{011}(r)\right]^{2}-\frac{1}{k T} u_{00 ; i s}^{000}(r),
$$

where $\rho_{2}=N_{2} / V$ is the number density of the salt, $c_{00 ; i s}^{000}(r)$ is the angle-independent projection of the ion-solvent direct correlation function and $k T$ is the Boltzmann constant times the absolute temperature. It immediately follows that $u_{i s}^{\Delta p}(R)$ may contribute to the low-concentration limiting behavior of $C_{i s}=\tilde{c}_{00 ; i s}^{000}(k=0)$ [cf. Eq. (34) of Ref. 29]. In order to determine if this is in fact the case we now examine the large- $R$ low-concentration dependence of the terms contributing to $\left\langle\Delta \mathbf{E}_{l}(R)\right\rangle$.

It can be shown ${ }^{1}$ that at $\rho_{2}=0$,

$$
h_{02 ; i s}^{022}(r) \rightarrow a / r^{3} \text { as } r \rightarrow \infty \text {, }
$$

where $a$ is a constant. If we insert this form for $h_{02 ; i s}^{022}(r)$ into Eq. (51) and integrate, we discover that the integral evaluates to zero. This clearly implies that at infinite dilution

$$
\left\langle\Delta E_{l Q}(R)\right\rangle=0 \text { as } R \rightarrow \infty .
$$

Therefore, $\left\langle\Delta \mathbf{E}_{l Q}(R)\right\rangle$ has no long-range "tail" [i.e., it becomes zero as soon as $h_{02 ; i s}^{022}(r)$ attains its large-r behavior], and consequently it cannot contribute to the long-range behavior of $u_{i s}^{\Delta p}(R)$ or the low-concentration limiting slope of $C_{i s}$.

We now turn our attention to $\left\langle\Delta \mathbf{E}_{l I}(R)\right\rangle$. From Eq. (B6) of I we have that as $r \rightarrow \infty$ and $\kappa \rightarrow 0$,

$$
g_{i j}(r) \rightarrow 1-\frac{q_{i} q_{j}}{\epsilon_{0} k T} \frac{e^{-\kappa r}}{r},
$$

where $\kappa$ is the usual Debye screening parameter given by

$$
\kappa=\left(\frac{4 \pi}{\epsilon_{0} k T} \sum_{i} \rho_{i} q_{i}^{2}\right)^{1 / 2}
$$

and $\epsilon_{0}$ is the pure solvent dielectric constant. We insert Eq. (55a) into Eq. (25) and expand and integrate (see Appen- dix B). Collecting terms, applying the small- $\kappa$ limit, and employing charge neutrality yields

$$
\left\langle\Delta E_{I I}(R)\right\rangle=\frac{\left|q_{i}\right|}{R^{2}} e^{-\kappa R}(1+\kappa R),
$$

which represents the large- $R$ low-concentration limiting behavior of $\left\langle\Delta \mathbf{E}_{I I}(R)\right\rangle$. We point out that at infinite dilution (i.e., $\kappa=0$ ) Eq. (56) becomes

$$
\left\langle\Delta E_{l I}(R)\right\rangle=\frac{\left|q_{i}\right|}{R^{2}},
$$

which is obviously consistent with Eqs. (10) and (11).

In considering the low-concentration long-range dependence of $\left\langle\Delta \mathbf{E}_{l D}(R)\right\rangle$ it is convenient to split $\left\langle\Delta \mathbf{E}_{l D}(R)\right\rangle$, and hence Eq. (35), into two separate contributions: $\left\langle\Delta \mathbf{E}_{l m}(R)\right\rangle$ due only to $\mathrm{m}_{e}$ and $\left\langle\Delta \mathbf{E}_{l p}(R)\right\rangle$ due to $\Delta \mathrm{p}(R)$. First, we examine $\left\langle\Delta \mathbf{E}_{l m}(R)\right\rangle$. Using the limiting behavior of $h_{00 ; i s}^{011}(r)$ [cf. Eq. (B4) of Ref. 29], it follows that as $\rho_{2} \rightarrow 0$ and $R \rightarrow \infty$,

$$
\begin{aligned}
\left\langle\Delta E_{l m}(R)\right\rangle \rightarrow & \frac{\left|q_{i}\right|}{8 d_{s}^{3} R^{2}} \frac{\epsilon_{0}-1}{\epsilon_{0}} \int_{R-d_{s}}^{R+d_{s}}(1+\kappa r) e^{-\kappa r} \\
& \times\left[r^{2}-2\left(R^{2}+d_{s}^{2}\right)+\frac{\left(R^{2}-d_{s}^{2}\right)^{2}}{r^{2}}\right] d r .
\end{aligned}
$$

If we perform the integration in Eq. (58) and collect terms (see Appendix B), then we can show that in the limit $\kappa \rightarrow 0$ and $R \rightarrow \infty$,

$$
\left\langle\Delta \mathbf{E}_{l m}(R)\right\rangle=\frac{-2\left(\epsilon_{0}-1\right)}{3 \epsilon_{0}} \frac{\left|q_{i}\right|}{R^{2}} e^{-\kappa R}(1+\kappa R) .
$$

We remark that Eqs. (56) and (59) are very similar. They predict that at low concentration and long range nearly twothirds of the ion field is canceled by the lateral dipole field due to $\mathrm{m}_{e}$. Of course, this will not be the case when $\epsilon_{0}$ is small. This clearly demonstrates the importance of the dielectic screening of the solvent and is an obvious indication of how poor an approximation Eqs. (1) are for aqueous electrolyte solutions.

In order to determine the long-range, low-concentration behavior of $\left\langle\Delta \mathbf{E}_{l p}(R)\right\rangle$ we must first know $\Delta p(R)$, but $\Delta p(R)$ is a function of $\left\langle\Delta E_{l}(R)\right\rangle$, which in turn depends upon $\left\langle\Delta E_{l p}(R)\right\rangle$. However, we can solve for $\left\langle\Delta \mathbf{E}_{l p}(R)\right\rangle$ iteratively. The expression for $\left\langle\Delta E_{l p}(R)\right\rangle$ in the limit $R \rightarrow \infty$,

$$
\begin{aligned}
\left\langle\Delta E_{l p}(R)\right\rangle= & \frac{\pi \rho_{s}}{2 d_{s}^{3} R^{2}} \int_{R-d_{s}}^{R+d_{s}} \Delta p(r) \\
& \times\left[\left(r^{2}+R^{2}-d_{s}^{2}\right)^{2}-(2 r R)^{2}\right] d r,
\end{aligned}
$$

follows directly from Eq. (35) and the fact that $g_{i s}(r)=1$ for large $r$. Using Eqs. (5) and (7) combined with the results 
from Eqs. (54), (56), and (59), we have that the $n$th approximation for $\Delta p(R)$ is given by

$$
\begin{aligned}
\Delta p^{\{n\}}(R)= & \alpha\left[\frac{\epsilon_{0}+2}{3 \epsilon_{0}} \frac{\left|q_{i}\right|}{R^{2}} e^{-\kappa R}(1+\kappa R)\right. \\
& \left.+\left\langle\Delta E_{\{p}^{\{n-1\}}(R)\right\rangle\right] .
\end{aligned}
$$

where $\left\langle\Delta E_{l p}^{\{n-1\}}(R)\right\rangle$ is the $(n-1)$ th estimate for $\left\langle\Delta E_{i p}(R)\right\rangle$. Then starting with $\left\langle\Delta E_{l p}^{\{0\}}(R)\right\rangle=0$ and iterating Eqs. (60) and (61), employing the explicit form for the integral given in Appendix B [cf. Eq. (B6)], it is possible to show that

$$
\begin{aligned}
\Delta p^{\{\infty\}}(R)= & \alpha\left[\frac{\epsilon_{0}+2}{3 \epsilon_{0}} \frac{\left|q_{i}\right|}{R^{2}} e^{-\kappa R}(1+\kappa R)\right] \\
& \times\left(1-\zeta+\zeta^{2}-\zeta^{3}+\cdots\right),
\end{aligned}
$$

where

$$
\zeta=8 \pi \alpha \rho_{s} / 3 \text {. }
$$

For $|\zeta|<1$ (i.e., for $\alpha \rho_{s}<3 / 8 \pi$ ) we recognize the last factor in Eq. (62a) as the Taylor-series expansion for $1 /(1+\xi)$. We note that for water at $25^{\circ} \mathrm{C}, \zeta=0.403$. Therefore, we can immediately write

$$
\left\langle\Delta E_{l}(R)\right\rangle=\frac{\epsilon_{0}+2}{\left(3+8 \pi \alpha \rho_{s}\right) \epsilon_{0}} \frac{\left|q_{i}\right|}{R^{2}} e^{-\kappa R}(1+\kappa R),
$$

which is, of course, only valid in the limits $\kappa \rightarrow 0$ and $R \rightarrow \infty$.

Let us now consider $\left\langle\Delta \mathbf{E}_{l}(R)\right\rangle$ for the special case when the solvent is polarizable but nonpolar. In such a case, $\left\langle\mathbf{E}_{l}\right\rangle=0$ and $\mathbf{m}^{\prime}=0$. Then by definition $\left\langle\Delta \mathbf{E}_{l m}(R)\right\rangle$ must also be zero [we recall that in Eq. (35), as elsewhere, we have replaced $m^{\prime}$ with $m_{e}$ ]. Using Eqs. (54), (56), and (60), together with Eqs. (5) and (7), we repeat the iterative scheme outlined above to show that for this system at large $R$

$$
\left\langle\Delta E_{l}(R)\right\rangle=\frac{3}{3+8 \pi \alpha \rho_{s}} \frac{\left|q_{i}\right|}{R^{2}} e^{-\kappa R}(1+\kappa R),
$$

where in the definition of $\kappa$ [cf. Eq. (55b)] $\epsilon_{0}$ is now the high-frequency dielectric constant usually denoted $\epsilon_{\infty}$. The Clausius-Mosotti relationship, ${ }^{22}$

$$
\frac{\epsilon_{\infty}-1}{\epsilon_{\infty}+2}=\frac{4}{3} \pi \alpha \rho_{s},
$$

can be substituted into Eq. (64a), and taking the limit $\rho_{2}=0$ gives the infinite dilution relationship

$$
\left\langle\Delta E_{l}(R)\right\rangle=\frac{\epsilon_{\infty}+2}{3 \epsilon_{\infty}} \frac{\left|q_{i}\right|}{R^{2}} .
$$

Pollock, Alder, and Pratt ${ }^{30}$ have also studied the screening of the charge of a single ion placed in a nonpolar polarizable solvent and have obtained precisely the same expression for the large- $R$ dependence of the average local electric field. Moreover, they found that continuum theory predicts a different result. When the two relationships were compared with values for the average local field at large $R$ determined from computer simulation, ${ }^{30}$ Eq. (65) gave essentially exact results while the continuum expression was accurate only for small values of $\alpha \rho_{s}$.

It is also interesting to examine the long-range behavior of $\left\langle\Delta \mathrm{E}_{l}(R)\right\rangle$ at infinite dilution when the solvent is polar but not polarizable. Taking $\alpha=0$ and $\kappa=0$ in Eq. (63), we immediately have that

$$
\left\langle\Delta E_{l}(R)\right\rangle=\frac{\epsilon_{0}+2}{3 \epsilon_{0}} \frac{\left|q_{i}\right|}{R^{2}} .
$$

Curiously, Eqs. (65) and (66) are equivalent in form, indicating that the field due to a single ion is screened to the same extent, the screening function $[(\epsilon+2) / 3 \epsilon]$ being a general result, in both solvents.

It is clear from Eqs. (7) and (63) that $\Delta p(R)$ has the same long-range, low-concentration dependence as $h_{00 ; i s}^{011}(r)$, and hence we would expect it to influence the limiting behavior of $C_{i s}$. Using Eqs. (7), (9b), (52), and (63) and the definition of $C_{i s}$ given by Eq. (34) of Ref. 29, it is possible to show after considerable manipulation that as $\rho_{2} \rightarrow 0$,

$$
C_{i s}^{\Delta \rho} \rightarrow C_{i s}^{\Delta \rho ; 0}-\frac{3 \pi \alpha}{k T}\left[\frac{q_{i}\left(\epsilon_{0}+2\right)}{\left(3+8 \pi \alpha \rho_{s}\right) \epsilon_{0}}\right]^{2} \kappa,
$$

where $C_{i s}^{\Delta \rho}$ is the contribution to $C_{i s}$ due to $u_{i s}^{\Delta \rho}(R)$ and the superscript 0 indicates the infinite dilution result. We now recall the exact low-concentration relationship [cf. Eq. (37a) of Ref. 29]

$$
\begin{aligned}
& \left(v_{+} C_{+s}+v_{-} C_{-s}\right) \\
& \quad=\left(v_{+} C_{+s}^{0}+v_{-} C_{-s}^{0}\right)+S_{c} \sqrt{\rho_{2}}+\cdots,
\end{aligned}
$$

where $v_{+}$and $v_{-}$are stoichiometric constants. For fluids of nonpolarizable particles the HNC equation can be used to provide an estimate for the total limiting slope, $S_{c}$ (see Appendix B of Ref. 29). If we define $S_{c}^{\Delta p}$ as being the contribution to $S_{c}$ due to $u_{i s}^{\Delta P}(R)$, then combining Eqs. (55b) and (67) yields

$$
S_{c}^{\Delta p}=\frac{-12 \pi \alpha A v^{3 / 2}\left(\epsilon_{0}+2\right)^{2}}{\left(3+8 \pi \alpha \rho_{s}\right)^{2} \epsilon_{0}},
$$

where $v=v_{+}+v_{-}$and

$$
A=\frac{\sqrt{\pi}}{2}\left(\frac{\left|q_{+} q_{-}\right|}{\epsilon_{0} k T}\right)^{3 / 2}
$$

It follows from Eq. (36) of Ref. 29 that if $u_{i s}^{\Delta p}(R)$ influences $S_{c}$, then it must also contribute to the low-concentration limiting behavior of $G_{+s}=G_{-s}$. Therefore, $u_{i s}^{\Delta P}(R)$ will affect the limiting slopes of all thermodynamic properties of electrolyte solutions which have a dependence upon ionsolvent correlations (i.e., $G_{+s}$ ) at low concentrations. An important example is $\vec{V}_{2}$, the partial molar volume of the salt [cf. Eqs. (49) of Ref. 29].

It is interesting to explore further $S_{c}^{\Delta p}$ for nonpolar polarizable solvents. In this case we use Eq. (64a) instead of Eq. (63) and follow the arguments outlined above to obtain

$$
S_{c}^{\Delta p}=-12 \pi \alpha A v^{3 / 2}\left(\frac{3}{3+8 \pi \alpha \rho_{s}}\right)^{2} \epsilon_{\infty},
$$

where $A$ is given by Eq. (69b) with $\epsilon_{0}$ again replaced by $\epsilon_{\infty}$. Now in Ref. 29 it is shown that at least for systems with effective pairwise additive potentials $S_{c}$ is related to the bulk dielectric constant of the pure solvent. Specifically, for a nonpolar polarizable model one has [cf. Eq. (56) of Ref. 29] 


$$
\frac{1}{\epsilon_{\infty}}\left(\frac{\partial \epsilon_{\infty}}{\partial \rho_{s}}\right)_{T}=\frac{-S_{c}^{\Delta p}}{3 A v^{3 / 2}}
$$

Combining Eqs. (70) and (71) gives

$$
\frac{1}{\epsilon_{\infty}^{2}}\left(\frac{\partial \epsilon_{\infty}}{\partial \rho_{s}}\right)_{T}=4 \pi \alpha\left(\frac{3}{3+8 \pi \alpha \rho_{s}}\right)^{2}
$$

which upon integration yields the Clausius-Mosotti formula (64b). It is gratifying to see that for the nonpolar polarizable solvent everything is self-consistent to the Clausius-Mosotti level.

\section{RESULTS AND DISCUSSION}

The reference HNC (RHNC) approximation was used to study the effect of the RDMF theory on model aqueous electrolyte solutions. The models employed in these RDMF/RHNC calculations have been previously studied ${ }^{17,19,20}$ with the SCMF/RHNC approximations and consist of hard-sphere ions immersed in a polarizable dipolar tetrahedral-quadrupolar solvent. The parameters for the water-like solvent and the ion diameters used are summarized in Tables I and II of I. It is helpful to recall here that the hard-sphere diameter of the solvent, $d_{s}$, was taken to be 2.8 $\AA$ and that the reduced ion diameters, $d_{i}^{*}=d_{i} / d_{s}$, were $0.84,1.08,1.16,1.28,1.44$, and 1.96 for $\mathrm{Na}^{+}, \mathrm{K}^{+}, \mathrm{Cl}^{-}$, $\mathrm{Cs}^{+}\left(\mathrm{Br}^{-}\right), \mathrm{I}^{-}$, and $\mathrm{M}^{+}$, respectively. We point out that $\mathbf{M}^{+}$is roughly similar in size to tetra-alkylammonium ions and that $\mathrm{Cs}^{+}$and $\mathrm{Br}^{-}$have the same diameter in the present model. A value ${ }^{31}$ of $1.444 \times 10^{-24} \mathrm{~cm}^{3}$ was employed for the average polarizability $\alpha$ of the water-like solvent. Solutions of $\mathrm{NaCl}, \mathrm{KCl}, \mathrm{CsI}$, and $\mathrm{MBr}$ were examined at infinite dilution, while calculations were performed for $\mathrm{KCl}$ and CsI at low concentrations, i.e., $<0.1 M$. All the sytems investigated were at $25^{\circ} \mathrm{C}$. The method of numerical solution of the RDMF/RHNC equations is essenitally that of the SCMF/ RHNC theory, as given in I. The numerical solution, which now consists of a set of $c_{\mu v, \alpha \beta}^{m n l}(r)$ together with $\Delta p(R)$ for each ion, is iterated until convergence is obtained.

As in our previous calculations at finite concentations, ${ }^{17}$ the effective permanent dipole moment of the solvent was taken to be that of the pure solvent. It was found that at the low concentrations examined here, the inclusion of the RDMF ion-solvent interaction had a negligible effect (e.g., $<0.1 \%$ at $0.1 M$ ) upon the average local electric field of the bulk.

First we consider the additional ion-solvent interaction, $u_{i s}^{\Delta p}(R)$, as determined from Eq. (9b). In Fig. 4 we have compared $u_{i s}^{\Delta p}(R)$ given by the RDMF theory of $\mathrm{Na}^{+}$at infinite dilution with the result obtained if we ignore all the lateral solvent fields [i.e., use Eq. (1c)]. It can be seen that the RDMF theory predicts a spherically symmetric ion-solvent interaction which is much smaller in magnitude than the direct ion term. Clearly, the lateral solvent fields are having a very large effect upon $u_{i s}^{\Delta p}(R)$, even at smaller separations. At contact we observe that the RDMF result for $u_{i s}^{\Delta p}(R)$ is about seven times smaller than the direct ion term. We remark that the contact value of $u_{i s}^{\Delta p}(R)$ for $\mathrm{Na}^{+}$corresponds to $\Delta p\left(R=d_{i s}\right)=0.38 \mathrm{D}$. In Fig. 4 we find that $-u_{i s}^{\Delta p}(R)$ drops rapidly from contact, reaching a minimum

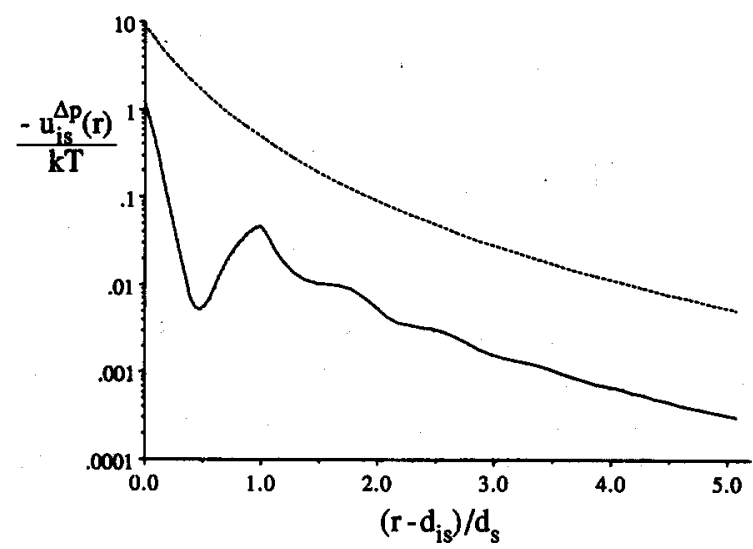

FIG. 4. Additional ion-solvent interaction due to $\Delta \mathrm{p}(R)$ for a $\mathrm{Na}^{+}$ion at infinite dilution. The solid curve is the RDMF result, while the dashed line represents $-u_{q p}(R) / k T$ as given by Eq. (1c). Note the semilog scale.

at a separation of approximately $0.5 d_{s}$, before increasing again to a local maximum at a separation of about one solvent diameter. Beyond a separation of one solvent diameter $u_{i s}^{\Delta \rho}(R)$ exhibits very little oscillatory behavior and runs essentially parallel to the $u_{q p}(R)$ curve, as can be seen from Fig. 4. For separations greater than $3 d_{s}$ the distance between the two curves corresponds to a factor of 17.0, which is also the numerical result given by the infinite dilution limit of $\mathrm{Eq}$. (63) combined with Eqs. (7) and (9) for the present waterlike solvent. For the other ions we have examined $u_{i s}^{\Delta p}(R)$ exhibits rather similar behavior. As one might expect, the oscillatory structure at smaller separations becomes less pronounced with increasing ion size.

In Fig. 5 we have compared SCMF/RHNC and RDMF/RHNC results for $g_{i s}(r)$ for $\mathrm{Na}^{+}$at infinite dilution. Clearly, the additional attraction between an ion and the surrounding solvent particles due to the RDMF increases the structure in $g_{i s}(r)$ for $\mathrm{Na}^{+}$. As can be seen from Fig. $5, g_{i s}(r)$ is most strongly influenced at short range, particularly at contact. Qualitatively similar results were found for all other ions considered. We also note that at infinite dilute $u_{i s}^{\Delta p}(R)$ makes a small but clearly evident contribution to $g_{i s}(r)$ at long range.

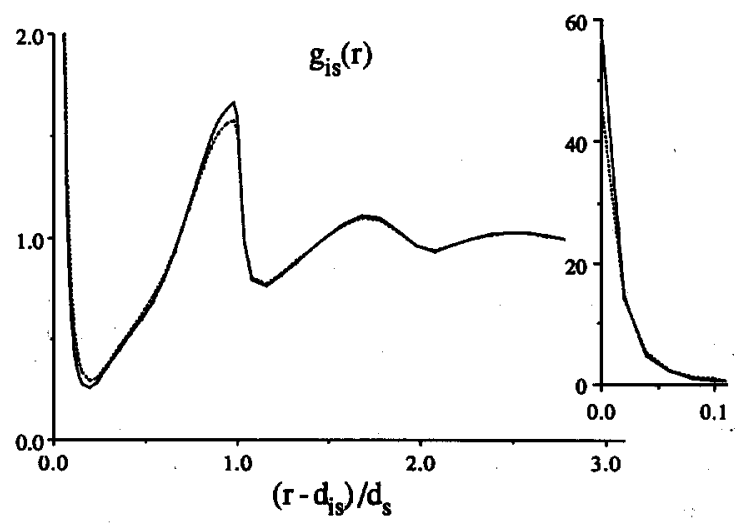

FIG. 5. Ion-solvent radial distribution functions for $\mathrm{Na}^{+}$at infinite dilution. The solid line is the present RDMF/RHNC result, while the dashed curve represents $g_{i s}(r)$ obtained from the SCMF/RHNC theory. 
In Fig. 6 we have shown the effect of $u_{i s}^{\Delta p}(R)$ upon unlike-ion potentials of mean force for $\mathrm{NaCl}$ and $\mathrm{MBr}$ at infinite dilution. In general, we find that the RDMF decreases the short-range attraction, which is consistent with the fact that the ion-solvent interaction has been increased. From Fig. 6(a) we observe that for $\mathrm{NaCl} w_{i j}(r)$ becomes much less attractive at small separations with the addition of $u_{i s}^{\Delta P}(R)$, although the well near the solvent separated distance has become somewhat deeper. At long range the RDMF/ RHNC and SCMF/RHNC curves become indistinguishable since the dielectric constant of the bulk solvent has remained unchanged. For a pair of larger ions, such as $\mathbf{M}^{+} /$ $\mathrm{Br}^{-}$, the addition of $u_{i s}^{\Delta p}(R)$ shifts $w_{i j}(r)$ to more positive values at all separations, as can be seen in Fig. $6($ b). In recent work using McMillan-Mayer level theory, Pettitt and Rossky ${ }^{32}$ have shown that some thermodynamic properties of model aqueous electrolyte solutions (in particular, the osmotic coefficients) are extremely sensitive to small changes in $w_{i j}(r)$. Hence, we would expect the shifts in $w_{i j}(r)$ observed here due to the RDMF for $\mathrm{NaCl}$ and $\mathrm{MBr}$ to result in significant changes in properties such as the mean activity coefficient for these solutions at finite concentra-
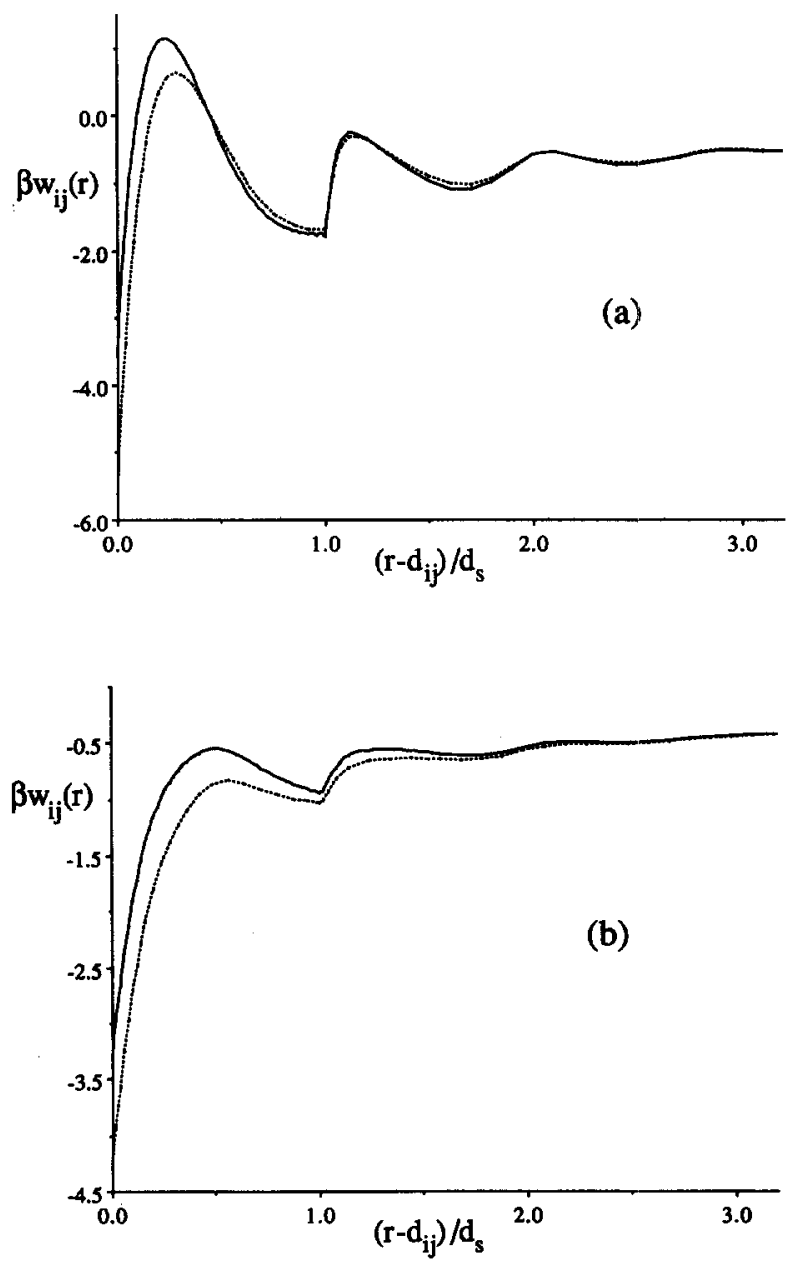

FIG. 6. Effect of the RDMF theory upon the unlike-ion potentials of mean force for (a) $\mathrm{Na}^{+} / \mathrm{Cl}^{-}$and (b) $\mathrm{M}^{+} / \mathrm{Br}^{-}$. The solid curves represent $\beta w_{i j}(r)$ when $u_{i s}^{\Delta p}(R)$ has been included in the ion-solvent potential, while the dashed lines are results obtained when the solvent polarizability is treated only at the SCMF level. tions. As discussed below this is indeed the case.

The influence of the RDMF upon the like-ion potentials of mean force at infinite dilution for $\mathrm{Na}^{+}$and $\mathrm{M}^{+}$is demonstrated in Fig. 7. Here we find that large and small ions show opposite effects. In Fig. 7(a) we see that for $\mathrm{Na}^{+}$the addition of $u_{i s}^{\Delta P}(R)$ shifts $w_{i i}(r)$ to more negative values at all separations. Clearly, the increased ion-solvent interaction has increased the probability of finding two $\mathrm{Na}^{+}$ions close together. Similar but less dramatic changes were found for $\mathrm{K}^{+}$and $\mathrm{Cl}^{-}$. For $\mathrm{M}^{+}$we observe from Fig. 7(b) that $w_{i i}(r)$ is shifted to more positive values at all separations with the inclusion of the RDMF. This behavior is consistent with the proposed ${ }^{17,19}$ hydrophobic nature of these large ions; the increased ion-solvent interaction reduces their hydrophobic nature and consequently decreases the probability of finding them at small separations. For $\mathrm{I}^{-} w_{i i}(r)$ exhibits similar but again less pronounced changes. We remark that for $\mathrm{Cs}^{+}$and $\mathrm{Br}^{-} w_{i i}(r)$ showed very little sensitivity to the presence of $u_{i s}^{\Delta p}(R)$.

In the derivation of the RDMF theory given above, it was shown that $u_{i s}^{\Delta p}(R)$ will contribute to the low-concentration behavior of $C_{i s}$ [cf. Eq. (67)] and an explicit expression [cf. Eqs. (69)] for $S_{c}^{\Delta p}$ was obtained. In Fig. 8 we have compared the low-concentration dependence of
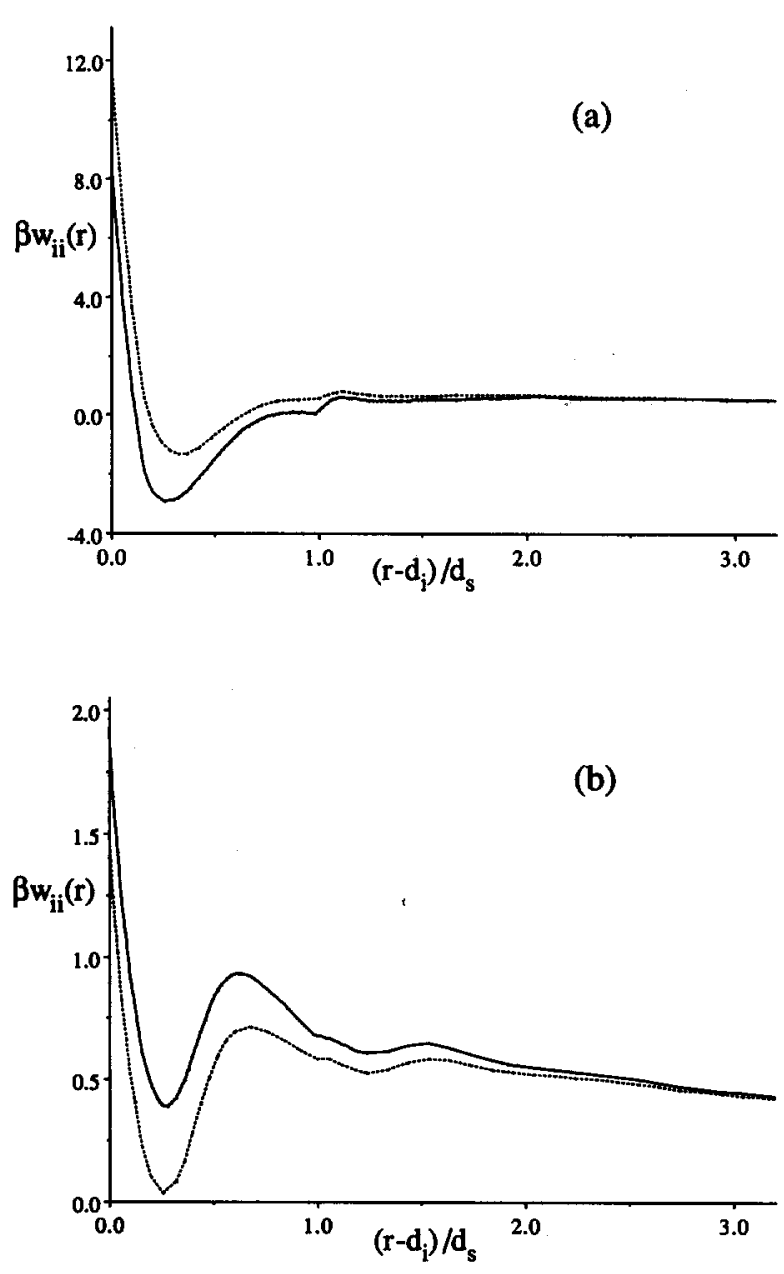

FIO. 7. Effect of the RDMF theory upon the like-ion potentials of mean force for (a) $\mathrm{Na}^{+} / \mathrm{Na}^{+}$and (b) $\mathrm{M}^{+} / \mathrm{M}^{+}$. The curves are as in Fig. 6 . 


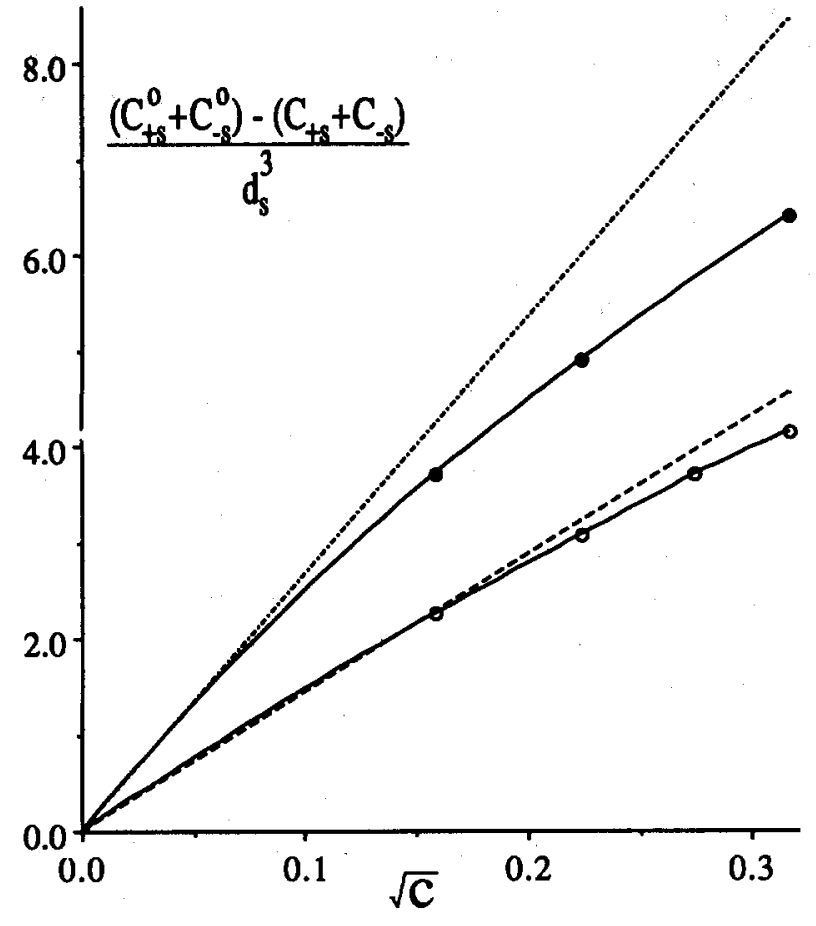

FIG. 8. Effect of the RMDF theory upon $\left(v_{+} C_{+s}+v_{-} C_{-s}\right)$. For ease of comparison we have plotted the difference between the function's infinite dilution values and $\left(v_{+} C_{+s}+v_{-} C_{-s}\right)$ itself. The open circles are SCMF/ RHNC results for model CsI solutions, while the solid circles represent values obtained when $u_{i s}^{\Delta p}(R)$ has been included. The dashed line is the limiting slope $S_{c}$ determined from Eq. (B6) of Ref. 29. The dash-dotted line represents the sum of $S_{c}^{\Delta p}$, given by Eqs. (69), and the result from Eq. (B6) of Ref. 29.

$\left(v_{+} C_{+s}+v_{-} C_{-s}\right)$ for model CsI solutions. At very low concentration the numerical results do approach their respective limiting laws. It is obvious from Fig. 8 that $S_{c}^{\Delta p}$ makes an appreciable contribution to the limiting slope of $\left(v_{+} C_{+s}+v_{-} C_{-s}\right)$, virtually doubling the total value. Clearly, $u_{i s}^{\Delta p}(R)$ can be expected to significantly affect the limiting behavior of all thermodynamic properties which depend upon ion-solvent correlations at low concentration. We point out that the infinite dilution values, $C_{i s}^{0}$, of $C_{i s}$ also increase substantially with the addition of $u_{i s}^{\Delta p}(R)$.

Model solutions of both $\mathrm{KCl}$ and CsI were studied at low concentration (i.e., for $c \leqslant 0.1 M$ ) using the RDMF/ RHNC theory. Comparison with previous SCMF/RHNC results ${ }^{17,19}$ reveals only slight changes in quantities such as the dielectric constant and the isothermal compressibility. As we would expect from our earlier discussion, the derivatives of the logarithm of the mean activity coefficient, $\left(\partial \ln \gamma_{ \pm} / \partial \rho_{2}\right)_{T, P}$ [cf. Eq. (56) of I], showed great sensitivity to the inclusion of $u_{i s}^{\Delta p}(R)$, even at the low concentrations examined. At $0.1 M$, the derivative for CsI was found to decrease in magnitude (i.e., become more positive) by more than $50 \%$, while for $\mathrm{KCl}$ the decrease was about $40 \%$. As a result, the values of $\left(\partial \ln \gamma_{ \pm} / \partial \rho_{2}\right)_{T, P}$ for both salts at $0.1 M$ were approximately half that given by the Debye-Hückel limiting law. We recall that in our previous SCMF/RHNC calculations the theoretical curves for $\ln \gamma_{ \pm}$deviated from their limiting law much more slowly, in general, than the experimental curves (cf. Fig. 10 of $I$ ). This is clearly not the case with the present RDMF/RHNC results for $\mathrm{KCl}$ and CsI which are in fact larger than the experimental values at least up to $0.1 M$.

The partial molar volumes of the solute, $\bar{V}_{2}$, also proved to be particularly sensitive to the addition of $u_{i s}^{\Delta p}(R)$ to the ion-solvent interaction. The infinite dilution values showed strong dependence. For example, for $\operatorname{CsI} \bar{V}_{2}^{0}=74.4 \mathrm{~cm}^{3}$ / mol within the SCMF/RHNC theory, and $40.1 \mathrm{~cm}^{3} / \mathrm{mol}$ with the RDMF included. Moreover, the limiting slope for $\bar{V}_{2}, S_{v}$, almost doubled due to a similar increase in $S_{c}$. Unfortunately, even if we were to use only $S_{c}^{\Delta p}$ in determining $S_{v}$ [cf. Eq. (52b) of I], we discover that this theoretical limiting slope for $\bar{V}_{2}$ still exceeds the true results for 1:1 aqueous electrolyte solutions at $25^{\circ} \mathrm{C}$ by more than a factor of 3 , even after correcting for the difference in the compressibilities of the present water-like solvent and real water (cf. Fig. 8 of I). In all likelihood this indicates ${ }^{33}$ that the RDMF theory overestimates the magnitude of $u_{i s}^{\Delta p}(R)$ at long range. We note that since $u_{i s}^{\Delta p}(R)$ depends upon the square of $\left\langle\Delta E_{l}(R)\right\rangle$, any error in $\left\langle\Delta E_{l}(R)\right\rangle$ will have a relatively large influence upon $C_{i s}$, and hence upon its limiting slope. For example, if we ignore dielectric screening and assume that $u_{i s}^{\Delta p}(R)=u_{q p}(R) e^{-\kappa R}(1+\kappa R)$, where $u_{q p}(R)$ is defined by Eq. (1c), the limiting slope would be about 17 times larger than the value we find. Since the theory contains a number of approximations, the precise origin of the remaining error in the magnitude of $u_{i s}^{\Delta P}(R)$ at large $R$ is not clear. We have shown above that at infinite dilution the RDMF result for the average local electric field at long range is equivalent (to Clausius-Mosotti level) to the expression of Pollock, Alder, and Pratt $^{30}$ for the special case when the solvent is polarizable but nonpolar [cf. Eq. (65) ]. A very similar expression [cf. Eq. (66)] was obtained for the case when the solvent is polar but nonpolarizable. However, the accuracy of the latter expression is not known since there are no computersimulation results available for this system.

\section{CONCLUSIONS}

In this article we have developed a new level of theory with which to study electrolyte solutions containing a polarizable solvent. The RDMF approximation allows us to examine the average local electric field experienced by a solvent molecule at a distance $R$ from an ion. It gives rise to an effective spherical potential between the ion and the solvent particles around it. This general formulation is then applied to a particular model for aqueous electrolyte solutions, one consisting of hard-sphere ions immersed in a polarizable hard-sphere water-like solvent with only dipole and tetrahedral-quadrupole moments. Explicit expressions are derived for electric field terms which take into account the lateral dipole and quadrupole moments of the surrounding solvent molecules, as well as all other ions in the system. We find that these terms can make a significant contribution to the average local electric field and tend to cancel the direct field due to the charge on the ion. Particular attention has been focused upon the long-range, low-concentration dependence of $\left\langle\Delta \mathrm{E}_{l}(R)\right\rangle$. We have shown that the resulting effective 
spherical potential will influence the low-concentration limiting behavior of those thermodynamic properties which depend upon ion-solvent corrections at low concentration.

RDMF/RHNC calculations were performed for several model aqueous 1:1 electrolytes at infinite dilution and at low concentration and compared with previous SCMF/ RHNC results ${ }^{17,19}$ at $25^{\circ} \mathrm{C}$. The electric field due to the ionic charge was found to be substantially reduced by the lateral solvent fields, even at small separations. The RDMF theory had a moderate effect upon the ion-solvent structure. However, its impact upon ion-ion correlations was generally much more significant, and thermodynamic properties such as the mean activity coefficient were observed to be rather sensitive to the presence of the $u_{i s}^{\Delta p}(R)$ potential. The addition of the effective ion-solvent interaction also had a strong influence upon $\bar{V}_{2}$, as we might expect.

We have shown that the RDMF theory can be used to obtain an accurate expression ${ }^{30}$ for the long-range screening of an ionic charge in the special case of a single ion immersed in a polarizable but nonpolar solvent. For other quantities the RDMF approximation is less satisfactory, even in the long-range and low-concentration limits. For example, the RDMF contribution to the limiting slope for $\bar{V}_{2}$ exceeds the known macroscopic result for real aqueous electrolyte solutions. Nevertheless, our calculations have clearly demonstrated that the spherically symmetric induced ion-solvent interactions considered here can have a significant impact upon the structural and thermodynamic properties of electrolyte solutions. These polarization effects clearly merit further study and it would be interesting to test the present RDMF formulation with careful computer simulations.

\section{ACKNOWLEDGMENTS}

We are grateful for the financial support of the Natural Sciences and Engineering Research Council of Canada.

\section{APPENDIX A: TRANSFORMATION OF $\Phi^{123}\left(\Omega_{1}, \Omega_{2}, \boldsymbol{f}\right)$}

We begin by expressing the unit vectors $\hat{\mathbf{x}}_{2}, \hat{\mathbf{y}}_{2}$, and $\hat{\mathbf{z}}_{2}$ in terms of the Euler angles $\alpha_{2}^{I}, \beta_{2}^{I}$, and $\gamma_{2}^{I}$ associated with the ion reference frame (see Appendix C of Ref. 28). In order to go from the ion reference frame to the frame $(\hat{\mathbf{x}}, \hat{\mathbf{y}}, \hat{\mathbf{z}})$, we must rotate about the $y_{I}$ axis by an angle $\omega$ [see Fig. 3(b)]. Therefore, we apply the rotation matrix ${ }^{27}$

$$
\mathbf{R}=\left[\begin{array}{ccc}
\cos \omega & 0 & -\sin \omega \\
0 & 1 & 0 \\
\sin \omega & 0 & \cos \omega
\end{array}\right]
$$

to the unit vectors $\hat{\mathbf{x}}_{2}, \hat{\mathbf{y}}_{2}$, and $\hat{\mathbf{z}}_{2}$. We can also express $\hat{\mathbf{x}}_{2}, \hat{\mathbf{y}}_{2}$, and $\hat{z}_{2}$ in terms of the Euler angles $\alpha_{2}, \beta_{2}$, and $\gamma_{2}$ (see Appendix Cof Ref. 28). Equating the components of the two representations for $\hat{\mathbf{x}}_{2}, \hat{\mathbf{y}}_{2}$, and $\hat{\mathbf{z}}_{2}$ and simplifying yields several relationships between the two sets of Euler angles associated with the two different reference frames. Explicitly, we have

$$
\begin{aligned}
& \sin \beta_{2}=\frac{\cos \alpha_{2}^{I} \cos \omega}{\cos \alpha_{2}}=\frac{\sin \alpha_{2}^{I}}{\sin \alpha_{2}}, \\
& \cos \beta_{2}=\cos \alpha_{2}^{I} \sin \omega,
\end{aligned}
$$

$$
\cos 2 \gamma_{2}=\cos 2 \gamma_{2}^{I} \sin ^{2} \alpha_{2}\left(\frac{\cos ^{2} \omega}{\sin ^{2} \alpha_{2}^{I}}-\sin ^{2} \omega\right),
$$

and

$$
\sin 2 \gamma_{2}=-2 \cos 2 \gamma_{2}^{I} \sin ^{2} \alpha_{2}\left(\frac{\sin \omega \cos \omega}{\sin \alpha_{2}^{I}}\right) .
$$

We substitute these expressions into Eq. (38b), for which we take $\alpha_{1}=0$ (this follows from our choice of reference frame), and after considerable manipulation and simplification (see Appendix C of Ref. 28) Eq. (44) can be obtained.

\section{APPENDIX B: A WORKED EXAMPLE OF AN EXPONENTIAL INTEGRAL}

We consider an integral of the general form [cf. Eqs. (60) and (61)]

$$
\begin{aligned}
F= & a \int_{R-d}^{R+d}(1+\kappa r) \frac{e^{-\kappa r}}{r^{2}}\left[\left(r^{2}+R^{2}-d^{2}\right)^{2}\right. \\
& \left.-(2 r R)^{2}\right] d r,
\end{aligned}
$$

where $a$ is some constant expression. It is the behavior of $F$ as $\kappa \rightarrow 0$ that is required. For convenience Eq. (B1) is rewritten in the form

$$
F=a\left(I_{3}+I_{2}+I_{1}+I_{0}+I_{-1}+I_{-2}\right),
$$

where

$$
\begin{aligned}
& I_{3}=\kappa \int_{R-d}^{R+d} r^{3} e^{-\kappa r} d r, \\
& I_{2}=\int_{R-d}^{R+d} r^{2} e^{-\kappa r} d r, \\
& I_{1}=-2 \kappa\left(R^{2}+d^{2}\right) \int_{R-d}^{R+d} r e^{-\kappa r} d r, \\
& I_{0}=-2\left(R^{2}+d^{2}\right) \int_{R-d}^{R+d} e^{-\kappa r} d r, \\
& I_{-1}=\kappa\left(R^{2}-d^{2}\right)^{2} \int_{R-d}^{R+d} r^{-1} e^{-\kappa r} d r,
\end{aligned}
$$

and

$$
I_{-2}=\left(R^{2}-d^{2}\right)^{2} \int_{R-d}^{R+d} r^{-2} e^{-\kappa r} d r .
$$

Using standard forms ${ }^{24}$ for the integrals in Eqs. (B2b)(B2e) and (B2g) and canceling terms whenever possible, yields

$$
\begin{aligned}
F= & a\left\{e ^ { - \kappa r } \left[\left(-r^{3}-\frac{4}{\kappa} r^{2}-\frac{8 r}{\kappa^{2}}-\frac{8}{\kappa^{3}}\right)\right.\right. \\
& \left.\left.+2\left(R^{2}+d^{2}\right)\left(r+\frac{2}{\kappa}\right)-\frac{1}{r}\left(R^{2}-d^{2}\right)^{2}\right]\right\}_{R-d}^{R+d} .
\end{aligned}
$$

If we then evaluate Eq. (B3) at its limits and rearrange we find that

$$
\begin{aligned}
F= & a e^{-\kappa R}\left[\left(\frac{8}{k^{3}}+\frac{8 R}{\kappa^{2}}\right)\left(e^{\kappa d}-e^{-\kappa d}\right)\right. \\
& \left.-\left(\frac{8 d}{k^{2}}+\frac{8 R d}{\kappa}\right)\left(e^{\kappa d}+e^{-\kappa d}\right)\right] .
\end{aligned}
$$

Now by expanding the exponentials, we can show that at small $\kappa$ 


$$
e^{\kappa d}-e^{-\kappa d}=2 \kappa d+\frac{1}{3} \kappa^{3} d^{3}
$$

and

$$
e^{\kappa d}+e^{-\kappa d}=2+\kappa^{2} d^{2}
$$

Finally, combining Eqs. (B4) and (B5) and simplifying, we obtain

$$
F=\frac{-16 d^{3}}{3} a(1+\kappa R) e^{-\kappa R}
$$

in the limit $\kappa \rightarrow 0$.

'G. Stell, G. N. Patey, and J. S. Høye, Adv. Chem. Phys. 38, 183 (1981) ${ }^{2}$ S. L. Carnie and G. N. Patey, Mol. Phys. 47, 1129 (1982).

${ }^{3}$ M. S. Wertheim, Annu. Rev. Phys. Chem. 30, 471 (1979).

${ }^{4}$ G. N. Patey and J. P. Valleau, Chem. Phys. Lett. 58, 157 (1978).

${ }^{5}$ V. Venkatasubramanian, K. E. Gubbins, C. G. Gray, and C. G. Joslin, Mol. Phys. 52, 1411 (1984).

${ }^{6}$ J. M. Caillol, D. Levesque, J. J. Weis, P. G. Kusalik, and G. N. Patey, Mol. Phys. 55, 65 (1985).

'G. N. Patey, G. M. Torrie, and J. P. Valleau, J. Chem. Phys. 71, 96 (1979)

${ }^{8}$ F. J. Vesley, Chem. Phys. Lett. 56, 390 (1978).

${ }^{9}$ E. L. Pollock, B. J. Alder, and G. N. Patey, Physica A 108, 14 (1981).

${ }^{10}$ S. Murad, Mol. Phys. 51, 525 (1984).

"J. R. Reimers, R. O. Watts, and M. L. Klein, Chem. Phys. 64, 95 (1982);

J. R. Reimers and R. O. Watts, ibid. 91, 201 (1984).

${ }^{12}$ P. Barnes, J. L. Finney, J. D. Nicholas, and J. E. Quinn, Nature (London) 282, 459 (1979).

${ }^{13}$ J. A. C. Rullmann and P. Th. Van Duijnen, Mol. Phys. 63, 451 (1988).
${ }^{14}$ M. Wojcik and E. Clementi, J. Chem. Phys. 84, 5970 (1986).

${ }^{15}$ R. W. Impey, P. A. Madden, and I. R. McDonald, J. Phys. Chem. 87, 5071 (1983)

${ }^{16}$ G. N. Patey, D. Levesque, and J. J. Weis, Mol. Phys. 57, 337 (1986).

${ }^{17}$ P. G. Kusalik and G. N. Patey, J. Chem. Phys. 88, 7715 (1988).

${ }^{18}$ P. G. Kusalik and G. N. Patey, Mol. Phys. 65, 1105 (1988).

${ }^{19}$ P. G. Kusalik and G. N. Patey, J. Chem. Phys. 89, 5843 (1988).

${ }^{20}$ P. G. Kusalik and G. N. Patey, J. Chem. Phys. 89, 7478 (1988).

${ }^{21}$ G. N. Patey and S. L. Carnie, J. Chem. Phys. 78, 5183 (1983); P. G. Kusalik and G. N. Patey, ibid. 79, 4468 (1983).

${ }^{22}$ C. F. J. Böttcher, Theory of Electric Polarization, 2nd ed. (Elsevier, Amsterdam, 1973).

${ }^{23}$ W. F. Murphy, J. Chem. Phys. 67, 5877 (1977).

${ }^{24}$ M. R. Spiegel, Mathematical Handbook of Formulas and Tables (McGraw-Hill, New York, 1968).

${ }^{25}$ R. T. Weidner and R. L. Sells, Elementary Classical Physics (Allyn and Bacon, Boston, 1973), Vol. 2.

${ }^{26}$ D. Levesque, J. J. Weis, and G. N. Patey, Mol. Phys. 51, 333 ( 1984); J. M. Caillol, D. Levesque, J. J. Weis, P. G. Kusalik, and G. N. Patey, ibid. 62 , 461 (1987).

${ }^{27}$ O. Steinhauser and H. Bertagnolli, Ber. Bunsenges. Phys. Chem. 85, 45 (1981).

${ }^{28}$ P. G. Kusalik, Ph.D. dissertation, University of British Columbia, 1987.

${ }^{29}$ P. G. Kusalik and G. N. Patey, J. Chem. Phys. 86, 5110 (1987).

${ }^{30}$ E. L. Pollock, B. J. Alder, and L. R. Pratt, Proc. Natl. Acad. Sci. U.S.A. 77, 49 (1980).

${ }^{3}$ D. Eisenberg and W. Kauzmann, The Structure and Properties of Water (Clarendon, Oxford, 1969).

${ }^{32}$ B. M. Pettit and P. J. Rossky, J. Chem. Phys. 84, 5836 (1986).

${ }^{33} \mathrm{We}$ are assuming here that it is only the dipole moments of the water molecules that influence the long-range low-concentration behavior of $\left\langle\Delta \mathbf{E}_{l}(R)\right\rangle$ and all higher order multipole moments make no contribution [cf. Eq. (54)], as is the case for the present water-like model. 